\title{
Single-particle Lagrangian statistics from direct numerical simulations of rotating-stratified turbulence
}

\author{
D. Buaria $\odot,{ }^{1, *}$ A. Pumir $\odot,{ }^{2,1}$ F. Feraco $\odot,{ }^{3,4}$ R. Marino, ${ }^{3}$ A. Pouquet, ${ }^{5}$ \\ D. Rosenberg, ${ }^{6}$ and L. Primavera ${ }^{4}$ \\ ${ }^{1}$ Max Planck Institute for Dynamics and Self-Organization, 37077 Göttingen, Germany \\ ${ }^{2}$ Laboratoire de Physique, ENS de Lyon, CNRS and Université de Lyon, 69007 Lyon, France \\ ${ }^{3}$ Laboratoire de Mécanique des Fluides et d'Acoustique, École Centrale de Lyon, CNRS, \\ Université Claude Bernard Lyon 1, INSA de Lyon, 69134 Écully, France \\ ${ }^{4}$ Dipartimento di Fisica, Universitá della Calabria, 87036, Arcavacata di Rende, Italy \\ ${ }^{5}$ Laboratory for Atmospheric and Space Physics, University of Colorado \\ and National Center for Atmospheric Research, Boulder, Colorado 80309, USA \\ ${ }^{6} 288$ Harper St., Louisville, Colorado 80027, USA
}

(Received 26 September 2019; accepted 11 May 2020; published 2 June 2020)

Geophysical fluid flows are predominantly turbulent and often strongly affected by the Earth's rotation, as well as by stable density stratification. Using direct numerical simulations of forced Boussinesq equations, we study the influence of these effects on the motion of fluid particles. We perform a detailed study of Lagrangian statistics of acceleration, velocity, and related quantities, focusing on cases where the frequencies associated with rotation and stratification (RaS), $f$ and $N$, respectively, are held at a fixed ratio $N / f=5$. The simulations are performed in a periodic domain, at Reynolds number $\operatorname{Re} \approx 4000$, and Froude number Fr in the range $0.03 \lesssim \mathrm{Fr} \lesssim 0.2$ (with Rossby number $\mathrm{Ro}=5 \mathrm{Fr}$ ). As the intensity of RaS increases, a sharp transition is observed between a regime dominated by eddies to a regime dominated by waves, which corresponds to $\mathrm{Fr} \lesssim 0.07$. For the given runs, this transition to a wave-dominated regime can also be seemingly described by simply comparing the timescales $1 / N$ and $\tau_{\eta}$, the latter being the Kolmogorov timescale based on the mean kinetic energy dissipation. Due to the known anisotropy induced by RaS, we consider separately the motion in the horizontal and vertical directions. In the regime $N \tau_{\eta}<1$, acceleration statistics exhibit well known characteristics of isotropic turbulence in both directions, such as probability density functions with wide tails and acceleration variance approximately scaling as per Kolmogorov's theory. In contrast for $N \tau_{\eta}>1$, they behave very differently, experiencing the direct influence of the imposed rotation and stratification. On the other hand, the Lagrangian velocity statistics exhibit visible anisotropy for all runs; nevertheless the degree of anisotropy becomes very strong in the regime $N \tau_{\eta}>1$. We observe that in the regime $N \tau_{\eta}<1$, rotation enhances the mean-square displacements in horizontal planes in the ballistic regime at short times but suppresses them in the diffusive regime at longer times. This suppression of the horizontal displacements becomes stronger in the regime $N \tau_{\eta}>1$, with no clear diffusive behavior. In contrast, the displacements in the vertical direction are always reduced. This inhibition

\footnotetext{
*dhawal.buaria@ds.mpg.de; Current address: New York University, NY 11201, USA, dhawal.buaria@nyu.edu.

Published by the American Physical Society under the terms of the Creative Commons Attribution 4.0 International license. Further distribution of this work must maintain attribution to the author(s) and the published article's title, journal citation, and DOI. Open access publication funded by the Max Planck Society.
} 
is extremely strong in the $N \tau_{\eta}>1$ regime, leading to a scenario where particles almost appear to be trapped in horizontal planes.

DOI: 10.1103/PhysRevFluids.5.064801

\section{INTRODUCTION}

Transport of material substances plays a crucial role in many geophysical processes $[1,2]$, e.g., dispersion of pollutants and contaminants [3,4], droplet dynamics in clouds [5], mixing of planktons and other biomatter in the oceans [6]. The Lagrangian viewpoint following the motion of fluid particles [7] or analogous entities such as Brownian or inertial particles [8,9] provides a natural description of such transport processes. Not only are most geophysical flows turbulent, they are also often strongly influenced by anisotropies due to effects such as rotation (Coriolis force) and stratification (buoyancy force) or presence of magnetic fields [10]. While particle dispersion has been studied extensively in isotropic turbulence, e.g., see Refs. [11-13], it has only recently started receiving attention in anisotropic flows. In particular, several studies have focused on flows considering either the effects of rotation [14-16] or stratification separately [17-19]. However, in many applications, adequately describing the observed flow physics necessitates examining the combined effects of rotation and stratification ( $\mathrm{RaS})$, e.g., in the southern abyssal oceans with particularly high mixing intensities [20-22].

Arguably the most challenging aspect of studying the combined effects of $\mathrm{RaS}$ in turbulence is the enormous range of spatial and temporal scales associated with such flows. In isotropic turbulence, typically the only governing parameter is the Reynolds number $(\mathrm{Re})$, which directly provides a measure of the range of scales in the flow. However, at least three additional parameters have to be considered in RaS turbulence, namely, the Rossby number (Ro) and the Froude number (Fr) which, respectively, measure the strength of RaS, and the Prandtl number (Pr) which is the ratio of the fluid viscosity to the thermal diffusivity (see Sec. II for precise definitions). This leads to consideration of additional length and timescales-beyond the already wide range existing in isotropic turbulence-and makes the flow dynamics far more involved. In particular, it has been recognized that the interaction between linear and nonlinear processes in such flows leads to a rich variety of complex behavior, such as spontaneous generation of helicity, dual cascading of energy and many more [23-25]. Advances in computing power in the past decade have allowed for significant progress in understanding such flows, although mostly from the Eulerian perspective [26-31].

In this work, utilizing direct numerical simulations (DNS) of Boussinesq equations, our objective is to investigate the dispersion of fluid particles in RaS turbulence. In particular, we focus on the motion of single individual particles. In view of the very large parameter space associated with RaS turbulence, we limit our investigation to the case where $\mathrm{Ro} / \mathrm{Fr}=5$, i.e., the strength of stratification is five times that of rotation, which is largely relevant to the southern abyssal oceans [20,21]. We use the same grid sizes for all cases, giving $\mathrm{Re} \approx 4000-5000$, and Froude numbers in the range $0.03 \lesssim$ $\mathrm{Fr} \lesssim 0.2$. This regime of Fr corresponds to the transitional regime, where both turbulent eddies and inertia-gravity waves are expected to play an important role [32]. As recently demonstrated in the case of purely stratified flows, the transition toward a wave dominated regime is accompanied by intermittent large-scale vertical drafts $[33,34]$. We show that the presence of additional rotation does not, in general, prevent the manifestation of this intermittency and thereby also investigate its effect on Lagrangian statistics.

In studying the motion of single particles, in the spirit of earlier studies [11-13], we investigate the properties of both their acceleration and velocity. The intrinsic anisotropy of the flow makes it necessary to distinguish throughout between motion in the horizontal plane and in the vertical direction, which are, respectively, perpendicular and parallel to the direction of imposed stratification (and also the axis of rotation). The anisotropy of acceleration and velocity reveal strikingly different properties. For acceleration statistics, which are reflective of small scales of turbulence, two 
distinct regimes are observed depending on the strength of imposed RaS. At weak or moderate RaS, the properties of acceleration are qualitatively similar to those documented in isotropic turbulence (with minor quantitative deviations), suggesting that the imposed anisotropy at large scales does not significantly affect the small-scales. In contrast, when RaS becomes very strong, acceleration statistics are significantly affected and exhibit striking differences between the horizontal and vertical motions. In the range of parameters considered here, we observe a sharp transition between the two regimes, which can be simply quantified with the ratio of the Kolmogorov timescale and the stratification timescale (which is simply the inverse of the Brunt-Väisäla frequency). The qualitative change in the statistical properties of acceleration occurs simultaneously with the appearance of intermittent bursts in the flow.

The Lagrangian velocity statistics, in contrast to acceleration, are always affected by the imposed $\mathrm{RaS}$, with the degree of anisotropy expectedly increasing with the strength of the imposed RaS. However, the transition observed for acceleration statistics is also visible in velocity statistics, resulting in even stronger anisotropy in velocity statistics when $\mathrm{RaS}$ is very strong. Accordingly, we characterize the integral timescales based on velocity autocorrelations and investigate the displacement of particles. The particles move ballistically at short times. At long times, the emergence of a diffusive regime is evident for runs with moderate RaS. However for strong RaS, the dispersion, particularly in vertical direction is strongly suppressed, qualitatively consistent with earlier works on purely stratified flows.

This paper is organized as follows. In Sec. II, we discuss our numerical methods. Essential features of the underlying Eulerian flow are discussed in Sec. III. The statistics of acceleration are presented in Sec. IV. Section V presents our results on the Lagrangian autocorrelation functions of velocity along with results on how particles are displaced by the flow. Finally, Sec. VI contains our concluding remarks.

\section{NUMERICAL METHOD AND DATABASE}

We simulate the Eulerian flow by numerically integrating the incompressible Boussinesq equations in a rotating frame, with constant solid body rotation rate $\Omega$ (and frequency $f=2 \Omega$ ) and gravity $g$ anti-aligned in the vertical $(z)$ direction:

$$
\begin{gathered}
\partial \mathbf{u} / \partial t+\mathbf{u} \cdot \nabla \mathbf{u}=-\nabla P-f \mathbf{e}_{z} \times \mathbf{u}-N \theta \mathbf{e}_{z}+v \nabla^{2} \mathbf{u}+\boldsymbol{\Phi} \\
\partial \theta / \partial t+\mathbf{u} \cdot \nabla \theta=N w+\kappa \nabla^{2} \mathbf{u} \\
\nabla \cdot \mathbf{u}=0 .
\end{gathered}
$$

Here, $\mathbf{u}=(u, v, w)$ is the velocity field, $\theta$ is the temperature fluctuation (in units of velocity), $P$ is the pressure normalized by the background density, $v$ is the kinematic viscosity, $\kappa$ is the thermal diffusivity, and $N$ is the Brunt-Väisäla frequency, which characterizes the strength of imposed stratification. We take $v=\kappa$, assuming the Prandtl number to be unity. A large-scale stochastic forcing term, $\boldsymbol{\Phi}$, is utilized to achieve and maintain a statistically stationary state. The forcing is random in time and isotropic in Fourier space, with the energy being injected in a spherical shell of wave numbers given by $2<|\mathbf{k}|<3$ [35], with the characteristic forcing length scale $L_{f}=2 \pi / 2.5$. While the use of isotropic forcing to simulate innately anisotropic flows may appear unphysical in the present context, earlier comparisons with a quasigeostrophic forcing have demonstrated that the precise nature of the forcing does not have any significant effect on the flow properties in the stationary state, at least in the regimes studied here $[24,28,32,36]$.

To characterize RaS, we introduce the dimensionless Rossby (Ro) and Froude (Fr) numbers defined as [10]

$$
\mathrm{Ro}=\frac{U}{L f}, \quad \mathrm{Fr}=\frac{U}{L N},
$$


where $L$ and $U$ are, respectively, the characteristic length and velocity scales of the large-eddies of the flow, defined as $L=L_{f}$, and $U=\left\langle|\mathbf{u}|^{2}\right\rangle^{1 / 2}$, the mean amplitude of the velocity fluctuations. An important parameter is the ratio $N / f(=\mathrm{Ro} / \mathrm{Fr})$, which measures the relative strength of RaS. As already mentioned, we maintain $N / f=5$ for all runs, as relevant in some oceanographic situations [20,21].

In addition, we define the following dimensionless numbers:

$$
\operatorname{Re}=\frac{U L}{v}, \quad R_{\mathrm{IB}}=\frac{\langle\epsilon\rangle}{v N^{2}}, \quad R_{\mathcal{B}}=\operatorname{ReFr}^{2}, \quad \operatorname{Ro}_{\omega}=\frac{\left\langle\omega^{2}\right\rangle^{1 / 2}}{f},
$$

where $\langle\epsilon\rangle$ is the mean dissipation rate of turbulent kinetic energy and $\left\langle\omega^{2}\right\rangle$ is the mean enstrophy density (with $\omega$ being the magnitude of vorticity). It is worth noting that in homogeneous turbulence, as considered in this work, the two are related as $\left\langle\omega^{2}\right\rangle=\langle\epsilon\rangle / \nu=1 / \tau_{\eta}^{2}$, where

$$
\tau_{\eta}=(\nu /\langle\epsilon\rangle)^{1 / 2}
$$

is the Kolmogorov timescale characterizing the motion of turbulent eddies [37]. Here, Re is the Reynolds number based on large scale. The parameter $R_{\mathrm{IB}}$, often referred to as the buoyancy Reynolds number, provides a measure of the relative importance of the waves induced by stratification with respect to turbulent eddies in the flow (or alternatively, the relative importance of waves and nonlinear processes); e.g., see Refs. [26,38]. This is evident by considering the Ozmidov length scale $\ell_{\mathrm{OZ}}=\left(\langle\epsilon\rangle / N^{3}\right)^{1 / 2}$, which characterizes the gravity waves and the Kolmogorov length scale, $\eta=\left(v^{3} /\langle\epsilon\rangle\right)^{1 / 4}$, which characterizes the dissipation scale of turbulence, thereby giving $R_{\mathrm{IB}}=\left(\ell_{\mathrm{OZ}} / \eta\right)^{4 / 3}$. Alternatively, $R_{\mathrm{IB}}$ can be written as the ratio of two timescales: $R_{\mathrm{IB}}=\left(T_{N} / \tau_{\eta}\right)^{2}$, where $T_{N}=1 / N$ is the stratification timescale (and $\tau_{\eta}$ is the Kolmogorov timescale), once again characterizing the relative importance of stratification to that of turbulent eddies. An alternative perspective is also offered by the asymptotic scaling analysis of Ref. [39], which suggests that $R_{\mathrm{IB}}$ can be interpreted as a replacement for the classical Reynolds number in strongly stratified turbulence-providing a relative measure of inertial and viscous forces. However, utilizing this interpretation in presence of rotation requires caution, since it invalidates the said asymptotic analysis (especially when rotation and stratification are comparable in strength).

A separate definition of the buoyancy Reynolds number [28,32] is given by the parameter $R_{\mathcal{B}}$. The two parameters are related as $R_{\mathrm{IB}}=\beta R_{\mathcal{B}}$, where $\beta=\langle\epsilon\rangle L / U^{3}$. In fully developed turbulence, $\beta$ is expected to be constant as a consequence of the dissipation anomaly [40]. However, in the regimes considered here, it can be shown that $\beta$ depends on the strength of stratification [32]. Hence, in our analysis, we utilize $R_{\mathrm{IB}}$ to quantify the relative strength of stratification and turbulence. The relevance of this choice will become evident as our results are presented in later sections. Finally, in addition to the Rossby number Ro based on large scales, it is useful to define the micro-Rossby number $\mathrm{Ro}_{\omega}$, which in contrast to $R_{\mathrm{IB}}$, measures the relative strength of small-scale turbulent motions to that of the imposed rotation. However, using $\langle\epsilon\rangle=v\left\langle\omega^{2}\right\rangle$ due to statistical homogeneity, $\mathrm{Ro}_{\omega}$ can be simply related to $R_{\mathrm{IB}}$ as $\mathrm{Ro}_{\omega}^{2} / R_{\mathrm{IB}}=(N / f)^{2}$. Since $N / f$ is held constant in this work, the parameters $\mathrm{Ro}_{\omega}$ and $R_{\mathrm{IB}}$ essentially provide the same information.

The database utilized in the current work, along with the main simulation parameters, is summarized in Table I. The simulations were carried out using the geophysical high-order suite for turbulence (GHOST) code, a versatile, highly parallelized, pseudospectral code, utilizing hybrid MPI-OpenMP programming model, with second-order explicit Runge-Kutta time stepping [41]. All runs correspond to a $(2 \pi)^{3}$ periodic domain with $512^{3}$ grid points. The parameters $N$ and $f$ are varied over a range of values keeping the ratio $N / f$ fixed at 5 . In addition, for a systematic comparison, we have also performed additional simulations by setting $N=0$ or $f=0$ or both. The simulation with $N=f=0$ (case 0 ) simply corresponds to homogeneous isotropic turbulence (HIT). All the runs reported were started from an initial condition, consisting of a few random modes in the velocity field, whereas the temperature field, $\theta$, was initialized to zero. The Boussinesq equations were integrated until a statistically stationary state was reached. For adequate statistical 
TABLE I. DNS database used in the current work, with corresponding simulation parameters. All rotatingstratified runs (cases 1-6) correspond to $N / f=5$. The cases 7 and 8 , respectively, with only stratification and only rotation, correspond to the $N$ and $f$ values of case 5 . The case 0 corresponds to homogeneous isotropic turbulence (HIT) with $N=f=0$. The mean dissipation of kinetic energy $\langle\epsilon\rangle$ was obtained by averaging over all the grid points and the time over which particles were tracked in the simulations. Other parameters are defined in Eqs. (4) and (5).

\begin{tabular}{lccccccccc}
\hline \hline Case & $0(\mathrm{HIT})$ & 1 & 2 & 3 & 4 & 5 & 6 & $7(\mathrm{~s})$ & $8(\mathrm{r})$ \\
\hline$N$ & 0 & 2.948 & 4.915 & 7.372 & 11.80 & 14.74 & 16.62 & 14.74 & 0 \\
$f$ & 0 & 0.5896 & 0.9830 & 1.474 & 2.360 & 2.948 & 3.320 & 0 & 2.948 \\
$\nu$ & 0.0015 & 0.001 & 0.001 & 0.001 & 0.001 & 0.001 & 0.001 & 0.001 & 0.001 \\
$\mathrm{Fr}$ & $\infty$ & 0.168 & 0.114 & 0.086 & 0.069 & 0.063 & 0.045 & 0.030 & $\infty$ \\
$\mathrm{Ro}$ & $\infty$ & 0.840 & 0.570 & 0.430 & 0.345 & 0.315 & 0.225 & $\infty$ & 0.140 \\
$\operatorname{Re}$ & 2379 & 3117 & 3537 & 3963 & 5199 & 5861 & 4744 & 3942 & 2645 \\
$R_{\mathcal{B}}$ & $\infty$ & 90.1 & 42.9 & 26.9 & 24.7 & 23.2 & 9.61 & 3.0 & $\infty$ \\
$\langle\epsilon\rangle$ & 0.375 & 0.198 & 0.182 & 0.146 & 0.059 & 0.049 & 0.027 & 0.050 & 0.065 \\
$R_{\mathrm{IB}}$ & $\infty$ & 22.8 & 7.53 & 2.69 & 0.422 & 0.226 & 0.096 & 0.229 & $\infty$ \\
$\operatorname{Ro}_{\omega}$ & $\infty$ & 23.9 & 13.7 & 8.22 & 3.25 & 2.38 & 1.55 & $\infty$ & 2.73 \\
\hline \hline
\end{tabular}

sampling, we considered a simulation period of at least $10-20 T_{E}$ in the stationary state for each case, where $T_{E}=L / U$ is the large eddy turnover time. (Some additional details are available in the Supplemental Material [42].)

In Table I, an important point to note is that the values of $R_{\mathcal{B}}$ and $R_{\mathrm{IB}}$ monotonically decrease as $N$ and $f$ are increased. Values of $N$ and $f$ higher than those shown are avoided, since the resulting regime would be completely dominated by waves [32]. The simulations with either $N=0$ or $f=0$ have their $f$ or $N$ value (respectively) corresponding to case 5 , with one of the largest $N$ and $f$ values. However, as evident from Table I the values of the Fr (and Ro) numbers corresponding to runs 5 and 7 (respectively, runs 5 and 8) differ significantly, despite identical values of the stratification (respectively, rotation rate). These variations are a consequence of our dynamical definition of $U$, which accounts for the subtle interplay between turbulence and RaS. In particular, they reflect the significant variation in $U$ itself, which is discussed in Sec. III. Importantly, we observe that the value of $R_{\mathrm{IB}}$ is lower than 0.5 for Runs $4-6$, as well as for the purely stratified run, 7, suggesting a dominant role of the waves in these runs. In contrast, $R_{\mathrm{IB}}>2$ in all other runs, possibly pointing to a prevalent role of the eddies. This leads to two qualitatively different behaviors, as demonstrated by our results in the following sections.

Finally, to obtain Lagrangian statistics, the fluid particles are tracked in time along with the Eulerian flow in the stationary state, according to the basic equation of motion,

$$
\frac{d \mathbf{x}^{+}(t)}{d t}=\mathbf{u}^{+}(t)=\mathbf{u}\left[\mathbf{x}^{+}(t), t\right]
$$

where the superscript + denotes a Lagrangian quantity and the fluid particle velocity is simply defined to be the Eulerian velocity field evaluated at the instantaneous particle position. For each run listed in Table I, we additionally tracked the motion of $1.5 \mathrm{M}$ particles $\left(\mathrm{M}=10^{6}\right)$, which are randomly distributed throughout the entire domain at the time of injection. The number of fluid particles are held constant for all cases, since given the same grid size and approximately similar Reynolds numbers, their sampling requirements are also approximately the same. Similar to the Eulerian grid, the particles are distributed among parallel processors and tracked in time together with the velocity field using a second-order Runge-Kutta scheme, and standard interpolation methods based on cubic splines [43,44]. 

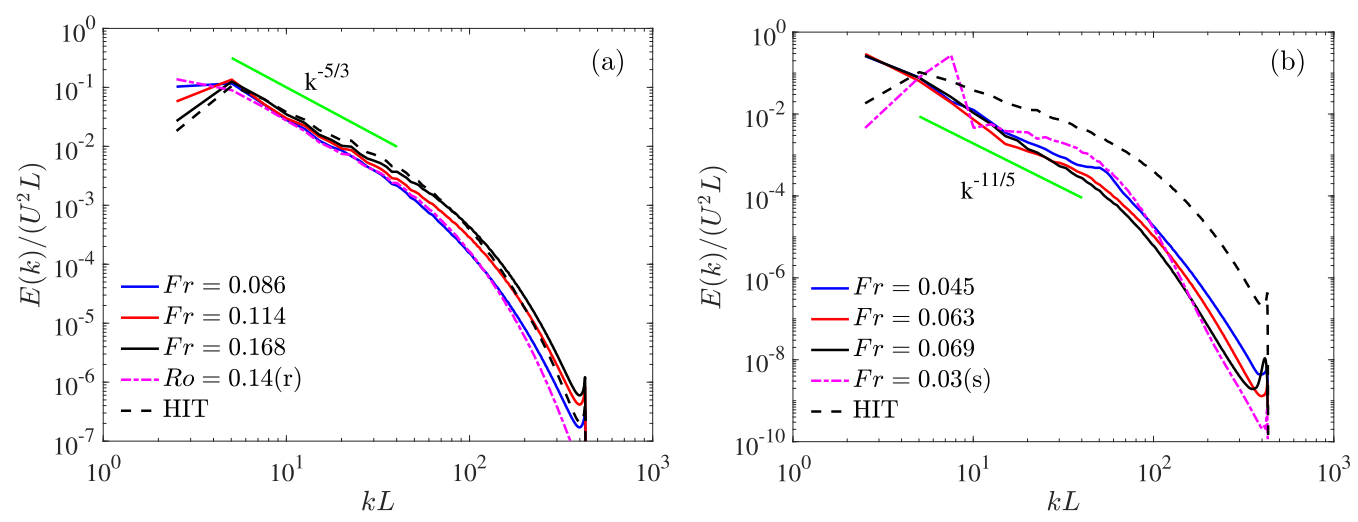

FIG. 1. The kinetic energy spectrum $E(k)$ normalized by $U^{2} L$ as a function of $k L$, where $U$ and $L$ are the large-scale velocity and length scales, respectively. The curves correspond to cases listed in Table I. Solid green lines indicating a spectral slope of $k^{-5 / 3}$ and $k^{-11 / 5}$ in panels (a) and (b), respectively, are drawn for reference.

\section{EULERIAN VELOCITY FIELD}

In this section, we provide a brief overview of the underlying Eulerian flow, which establishes the necessary framework for better understanding the Lagrangian statistics reported subsequently.

\section{A. Energy spectra}

We begin by considering the kinetic energy spectra, as shown in Fig. 1, which is one of the most common descriptor of Eulerian dynamics in turbulent flows [10]. The curves are normalized using the large-scale variables $U$ and $L$, and split into two subfigures depending on the strength of weak rotation and stratification $(\mathrm{RaS})$. Figure 1(a) shows the spectra for the runs with relatively weak $\mathrm{RaS}$, corresponding to $\mathrm{Fr} \geqslant 0.086$ and $R_{\mathrm{IB}}>1$, including the HIT and rotation-only runs. The main observation is that all the spectra demonstrate an approximate $k^{-5 / 3}$ Kolmogorov-like scaling in an intermediate range of $k$. This expectation is justified for the HIT run, as well as the $R_{\mathrm{IB}}>1$ runs, for which there is a sufficient scale separation between $\ell_{\mathrm{OZ}}$ and $\eta$ (note $R_{\mathrm{IB}}=\left(\ell_{\mathrm{OZ}} / \eta\right)^{4 / 3}$ as discussed earlier), to recover Kolmogorov-like turbulence [39,45]; although systematic deviations from the scaling are clearly evident as $R_{\mathrm{IB}}$ decreases. In fact, as shown later in this section, the large scales for $R_{\mathrm{IB}}>1$ runs are still predominantly anisotropic, though their impact on small-scales is weak.

However, in Fig. 1(b), the spectra for runs with stronger stratification, corresponding to $\mathrm{Fr} \leqslant$ 0.069 and $R_{\mathrm{IB}}<1$ are shown. The spectrum for the HIT run (dashed black line) is also included for comparison. As evident, this regime significantly differs from that at weaker stratification, with the spectra exhibiting a spectral slope steeper than $k^{-5 / 3}$ (since $R_{\mathrm{IB}}<1$ and hence $\ell_{\mathrm{OZ}}<\eta$ precludes Kolmogorov's theory from being applicable). It has been observed in the closely related context of a time-dependent flow [28,46] that the spectra followed the Bolgiano-Obhukov prediction $[47,48]$. In this regime, energy transfer is strongly affected by the temperature fluctuations (potential energy). The straight line in Fig. 1(b) indicates the corresponding $k^{-11 / 5}$ prediction. Given the small range of spatial scales available in our simulations, however, it is difficult to conclusively demonstrate that the spectra shown indeed conform to a $k^{-11 / 5}$ scaling (and the fact that the precise exponent can depend on the Froude number [49]). Nevertheless, the change in spectral slopes clearly evidences that the energy transfer between scales is strongly perturbed in runs with $R_{\mathrm{IB}}<1$, and much less so in runs with $R_{\mathrm{IB}}>1$. In fact, this demarcation based on $R_{\mathrm{IB}}$ becomes increasingly prominent as more results are discussed in later sections. The spectra of the temperature fluctuations (not shown) follow similar trends as that of velocity. 
TABLE II. Variance and kurtosis of velocity components. We use $\xi^{u}=\left\langle u_{\perp}^{2}\right\rangle^{1 / 2} /\left\langle u_{\|}^{2}\right\rangle^{1 / 2}$ to measure the anisotropy, whereas $K_{u_{\|}}$and $K_{u_{\perp}}$ are the kurtosis of $u_{\|}$and $u_{\perp}$, respectively. The skewness of these components (not shown) is consistent with 0 . The value(s) of kurtosis marked with an asterisk correspond to relatively low accuracy.

\begin{tabular}{lccccccccc}
\hline \hline Case & 0 & 1 & 2 & 3 & 4 & 5 & 6 & 7 & 8 \\
\hline Fr & $\infty$ & 0.168 & 0.114 & 0.086 & 0.069 & 0.063 & 0.045 & 0.030 & $\infty$ \\
Ro & $\infty$ & 0.840 & 0.570 & 0.430 & 0.345 & 0.315 & 0.225 & $\infty$ & 0.140 \\
$\left\langle u_{\perp}^{2}\right\rangle$ & 0.68 & 0.62 & 0.92 & 1.2 & 2.2 & 2.8 & 1.7 & 0.55 & 0.39 \\
$\left\langle u_{\|}^{2}\right\rangle$ & 0.67 & 0.28 & 0.17 & 0.097 & 0.022 & 0.019 & 0.005 & 0.0077 & 0.28 \\
$\xi^{\mu}$ & 1.01 & 1.48 & 2.33 & 3.52 & 10.0 & 12.0 & 18.4 & 8.4 & 1.18 \\
$K_{u_{\perp}}$ & 2.9 & 2.9 & 2.9 & 2.9 & 3.1 & 3.4 & 3.05 & 2.5 & 3.1 \\
$K_{u_{\|}}$ & 2.9 & 3.3 & 3.8 & 4.7 & 6.4 & 8.4 & $10.9 *$ & 3.2 & 3.1 \\
\hline \hline
\end{tabular}

\section{B. Anisotropy of velocity fluctuations}

The above transition between strong hydrodynamic turbulence and regimes dominated by RaS, as evidenced from the energy spectra, clearly warrant further inspection. An important aspect of the flows in this study is the existence of a strong anisotropy of velocity fluctuations. Namely, the presence of RaS induces very different properties of the velocity fluctuations in the horizontal plane and in the vertical direction. This anisotropy has been investigated in many earlier studies, in particular by analyzing the longitudinal and transverse energy spectra [10,28,39]. In the present work, we limit our discussion to the velocity components $u_{\perp}$ and $u_{\|}$, respectively, in the horizontal and vertical directions (perpendicular and parallel to gravity). The statistics involving $u_{\perp}$ are obtained by averaging the two components in the $x$ - $y$ horizontal plane (which is also the plane of rotation), whereas $u_{\|}$simply corresponds to the vertical $z$ component. An obvious manifestation of the anisotropy between the two directions is in variances of $u_{\perp}$ and $u_{\|}$, as shown in Table II. It is evident that apart from the HIT run (case 0) and to a large extent the rotation only run (case 8), the variances for both components are very different. To precisely quantify the anisotropy, we consider the ratio $\xi^{u}=\left[\left\langle u_{\perp}^{2}\right\rangle /\left\langle u_{\|}^{2}\right\rangle\right]^{1 / 2}$, also shown in Table II. Expectedly, this ratio is unity for case 0 and very close to unity for case 8 . However, for all runs with stratification, the ratio is always greater than 1 . For cases 1-3, the ratio increases slowly with decreasing Fr, whereas a sharp increase occurs for cases 4-6 (and also case 7). Clearly, this transition corresponds to the change in spectral slope observed for the spectra in Fig. 1. The runs with smaller $\xi^{u}$ correspond to spectra which show $k^{-5 / 3}$ scaling, whereas runs with sharp increase of $\xi^{u}$ correspond to spectra with steeper Bolgiano-like scaling. In these flows, the fluctuations of $u_{\|}$are essentially suppressed when the imposed RaS are strong. We note that the values shown here are consistent with the results of some earlier runs [32].

To further investigate the anisotropy, we next consider higher-order moments of the velocity components. Since the probability density functions (PDFs) of individual velocity components are symmetric (due to underlying symmetry of Boussinesq equations), we note that the values of the third moments of the velocity fluctuations are expected to be zero. Our results, not shown here, are compatible with this. Thus, we consider the fourth-order moment, through the kurtosis, defined as $K_{u_{i}}=\left\langle u_{i}^{4}\right\rangle /\left\langle u_{i}^{2}\right\rangle^{2}$. It is known that the PDFs of individual velocity components in HIT are approximately Gaussian, which implies that the kurtosis is approximately 3. Consistent with that, we find that the value of the kurtosis is very close to 3 in the case HIT (see Table II). In the case of a purely stratified flow, however, it was found that the distribution of the velocity component parallel to the direction of stratification, $u_{\|}$, could be significantly wider than Gaussian $[33,34]$. The strong deviations from a Gaussian distribution have been shown to originate from intermittent vertical drafts [34]. On the other hand, the kurtosis of $u_{\perp}$ is always found to be approximately 3 . Our values, as shown in Table II, are consistent with these trends. We find that $K_{u_{\perp}}$ is slightly smaller 
TABLE III. Variance and kurtosis of acceleration components, perpendicular and parallel to the axis of rotation/stratification. $\xi^{a}=\left[\left\langle a_{\perp}^{2}\right\rangle /\left\langle a_{\|}^{2}\right\rangle\right]^{1 / 2}$ measures the anisotropy, whereas the kurtosis, $K_{a_{\perp}}$ and $K_{a_{\|}}$, characterizes the extent of the PDFs (see Fig. 3). The underlying symmetry of the problem imposes that the skewness (not shown) is zero. The values of kurtosis marked with an asterisk correspond to relatively low accuracy.

\begin{tabular}{lccccccccc}
\hline \hline Case & 0 & 1 & 2 & 3 & 4 & 5 & 6 & 7 & 8 \\
\hline Fr & $\infty$ & 0.168 & 0.114 & 0.086 & 0.069 & 0.063 & 0.045 & 0.030 & $\infty$ \\
Ro & $\infty$ & 0.840 & 0.570 & 0.430 & 0.345 & 0.315 & 0.225 & $\infty$ & 0.140 \\
$\left\langle a_{\perp}^{2}\right\rangle$ & 16.4 & 6.9 & 6.3 & 5.4 & 5.4 & 9.3 & 6.21 & 0.48 & 2.1 \\
$\left\langle a_{\|}^{2}\right\rangle$ & 16.3 & 8.9 & 8.6 & 6.7 & 1.9 & 2.0 & 0.43 & 1.1 & 1.9 \\
$\xi^{a}$ & 1.01 & 0.88 & 0.85 & 0.90 & 1.67 & 2.11 & 3.80 & 0.66 & 1.05 \\
$K_{a_{\perp}}$ & 19.3 & 29.8 & 37.1 & $46.9^{*}$ & 6.52 & 7.50 & 14.3 & 4.38 & 22.8 \\
$K_{a_{\|}}$ & 19.6 & 22.3 & 27.2 & $40.5^{*}$ & 17.9 & 11.8 & 16.2 & 3.27 & $35.6^{*}$ \\
$\xi^{u} / \xi^{a}$ & 1.0 & 1.68 & 2.74 & 3.9 & 6.0 & 5.7 & 4.8 & 12.7 & 1.1 \\
\hline \hline
\end{tabular}

than 3 at low stratification, and slightly larger than 3 for runs with strong stratification (Fr $\lesssim 0.07$ ), with PDFs (not shown) being approximately Gaussian. In the vertical direction, $K_{u_{\|}}$increases very significantly beyond 3 as strength of $\mathrm{RaS}$ increases.

It is worthwhile to compare the dependence of $K_{u_{\|}}$in our RaS runs with the stratification-only runs reported in Ref. [34] (see in particular their Fig. 5). While the sharp transition in $K_{u_{\|}}$appears to be qualitatively similar, quantitatively the large values of $K_{u_{\|}}$for RaS runs occur at slightly lower Fr values than in Ref. [34]. For even smaller Fr (not considered in this work), in a wave-dominated regime, one can expect that $K_{u_{\|}}$for RaS runs will again become 3 [34], as it was the case for the stratification-only run (case 7) discussed here. Additional discussion of $K_{u_{\|}}$will be presented in the following section, in relation with acceleration statistics.

\section{ACCELERATION STATISTICS}

The acceleration experienced by a fluid element, defined by the rate of change of velocity in the Lagrangian frame, i.e., $\mathbf{a}^{+}=d \mathbf{u}^{+} / d t$ [where $\mathbf{u}^{+}$is defined in Eq. (7)] and resulting from the balance of forces acting on it, is arguably the simplest descriptor of its motion, as also directly reflected in the governing fluid equations. In addition to its fundamental importance in turbulence theory [13], a key motivation to study acceleration comes from its central role in stochastic modeling of turbulent dispersion [50]. In the following subsections, we study different aspects related to acceleration, namely, acceleration variance and kurtosis, the probability density functions and Lagrangian autocorrelations and frequency spectra. As already mentioned, the anisotropy of the flow makes it necessary to distinguish between the horizontal and vertical components of acceleration, denoted by $a_{\perp}^{+}$and $a_{\|}^{+}$, respectively. In this section, we demonstrate that the anisotropy properties of the acceleration components strongly differ from those of the velocity discussed in the previous section. For convenience, we will henceforth omit the superscript "+" from our notation, since our subsequent results only involve Lagrangian quantities.

\section{A. Acceleration variance}

Extending the earlier analysis of the Eulerian velocity field, we first examine the anisotropy in acceleration by considering the second- and fourth-order moments, namely, the variance and kurtosis, respectively. The moments are listed in Table III. Due to the underlying symmetry of the Boussinesq equations, the third and all other odd moments of acceleration components are zero (similar to the velocity components). As can been seen from cases 1-6, the properties of $a_{\perp}$ and $a_{\|}$ 
show striking differences. While the variances of $a_{\|}$rapidly decrease with decreasing Ro (and Fr), the variances of $a_{\perp}$ do not change that much. To better understand this behavior, we consider the anisotropy ratio $\xi^{a}=\left[\left\langle a_{\perp}^{2}\right\rangle /\left\langle a_{\|}^{2}\right\rangle\right]^{1 / 2}$. For the HIT run (case 0), the ratio, as expected, is equal to 1 , whereas for rot-strat cases two separate behaviors are visible.

The first corresponds to cases 1-3 with relatively weak rotation and stratification (RaS), where the ratio $\xi^{a}$ is approximately constant, and slightly smaller than 1 . The ratio being close to unity suggests that the effect of anisotropy on small scales is only minor. Furthermore, a simple explanation for the ratio slightly smaller than unity can be provided based on the knowledge that the relative strength of stratification is significantly stronger compared to that of rotation (since $N / f=5$ ). For that reason, the acceleration variance in the vertical direction is likely to be slightly more enhanced (due to stratification) than that in the horizontal direction (due to rotation). In contrast, runs 4-6 correspond to significantly stronger RaS, which leads to a different behavior, with $\xi^{a}>1$ and further increasing with decreasing Fr (and Ro). Since runs 4-6 correspond to $R_{\mathrm{IB}}<1$, they are in a regime where waves play a stronger role, compared to (turbulent) eddies. With strong stratification, the motion in the vertical direction is strongly suppressed, as also reflected in $\xi^{u}$ values (see Table II). With relative weaker effect of rotation, the acceleration in the horizontal direction is still affected by turbulent eddies, resulting in $\xi^{a}$ to become greater than 1 . Thereafter, the effect becomes even more pronounced with further increase in strength of RaS.

It is instructive to compare the acceleration variances for case 5 (with strong $\mathrm{RaS}$ ), to case 7 , with exactly the same stratification, $N$, but no rotation and to case 8 , with the same rotation, $f$, but no stratification. The variances of $a_{\perp}$ and $a_{\|}$are stronger in the presence of both RaS, than with either of the two effects taken separately. The anisotropy ratio $\xi^{a}$ also shows a very striking difference, when comparing runs 5,7 , and 8 . For rotation-only (run 8 ), we observe $\xi^{a} \approx 1$, implying little to no small-scale anisotropy. This can be viewed as consistent with the observation that the flow spectrum for run 8 is overall comparable to the Kolmogorov spectrum [see Fig. 1(a)], so the flow is dominated by eddies. On the other hand, the anisotropy ratio is $\xi^{a}=0.66<1$ for run 7 , implying stronger acceleration variances in the vertical than in the horizontal direction, but an opposite situation in the presence of both RaS: $\xi^{a}=2.1>1$. This difference can be traced back to the much larger value of $\left\langle a_{\perp}^{2}\right\rangle$ in the presence of rotation, a consequence of the enhancement of the horizontal component of acceleration due to the Coriolis force.

In comparing the anisotropy of acceleration components with that of velocity (see Table II), we find that $\xi_{a}$ is always smaller than $\xi_{u}$, and additionally very close to unity when $R_{\mathrm{IB}}>1$. This can be explained by realizing that velocity is sensitive to the large scales of the flow and is directly influenced by the imposed anisotropy. However, acceleration mostly samples the small scales, and is not likely sensitive to the imposed anisotropy (at large scales), provided the scale separation between the large and small scales is sufficiently wide-consistent with the notion of local isotropy as dictated by Kolmogorov's theory [7]. In the current scenario, this is realized when $R_{\mathrm{IB}}>1$, resulting in $\xi^{a}$ being nearly unity (and also a $k^{-5 / 3}$ range in the intermediate $k$ range as demonstrated earlier). Whereas for $R_{\mathrm{IB}}<1$, local isotropy is clearly violated, but still the anisotropy of acceleration is significantly weaker than that of velocity.

Additional insight can be obtained by considering the ratio $\xi^{u} / \xi^{a}$, also shown in Table III. For HIT, this ratio is unity, but with increasing strength of RaS, we observe $\xi_{u} / \xi_{a}$ increases, peaking at around 6 for case 4, and thereafter decreasing slightly with further increase in strength of RaS. A limiting value can be obtained in the case where RaS is very strong, such that the flow is dominated by linear processes (with turbulent eddies playing a very weak role). This would lead to $\xi^{u} / \xi^{a} \approx$ $N / f$, by assuming that $a_{\perp} \sim u_{\perp} f$ and $a_{\|} \sim u_{\|} N$. The value for case 6 , with $\xi^{u} / \xi^{a}=18.4 / 3.8 \approx 5$, appears to be consistent with this consideration, though it remains to be further tested for different $N / f$ values.

Given that the statistics of acceleration appear to conform with local isotropy for $R_{\mathrm{IB}}>1$, we consider the following relation from classical turbulence:

$$
\left\langle a^{2}\right\rangle=a_{0}\langle\epsilon\rangle^{3 / 2} v^{-1 / 2},
$$




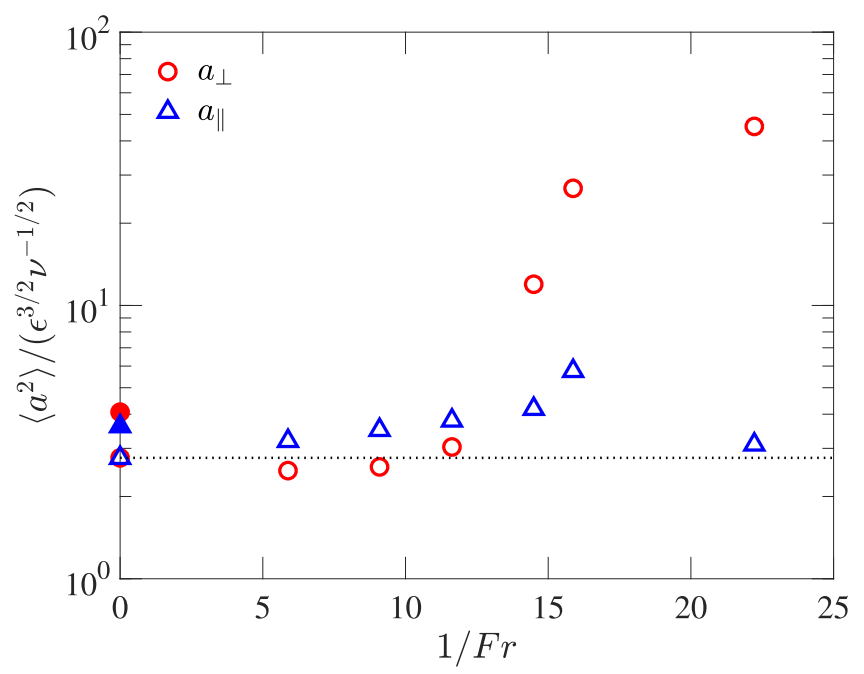

FIG. 2. Kolmogorov-scaled acceleration variance, as defined by Eq. (8), plotted as a function of 1/Fr. The parallel (vertical) and perpendicular (horizontal) components of accelerations are shown in blue triangles and red circles, respectively. The HIT run corresponds to $1 / \mathrm{Fr}=0$. In addition the rotation-only run also corresponds to $1 / \mathrm{Fr}=0$, but is shown in solid symbols.

which results from the application of Kolmogorov's similarity theory to acceleration variance [7]. Here, $a_{0}$ is a dimensionless constant, which is plausibly universal for HIT. Empirical evidence based on studies of isotropic turbulence has shown that $a_{0}$ increases slowly with Reynolds numbers [51], which can be possibly viewed as a manifestation of small-scale intermittency, unaccounted for in Kolmogorov's 1941 theory. In contrast, for RaS turbulence, one may expect additional deviations because of the imposed anisotropy, especially if Re is not very large.

Figure 2 shows the values of the $a_{0}^{\perp}$ and $a_{0}^{\|}$as a function of Fr for cases 1 through 6 , together with the value obtained for case 0 , in the absence of RaS. The values of $a_{0}^{\|}$and $a_{0}^{\perp}$ do not vary very much for $1 / \mathrm{Fr} \gtrsim 12.5$, and sharply increase, especially in the perpendicular direction, at large values of $1 /$ Fr. In particular, the value of $a_{0}^{\perp}$ at $1 / \mathrm{Fr} \approx 16$ exceeds that in the HIT case by a factor larger than $\sim 10$. This points to a strong difference of the small-scale properties between runs 4-6 and the HIT run (case 0$)$. In comparison, and despite the larger value of $N(N / f=5$ for runs 1-6), the value of $a_{0}^{\|}$remains comparable to that obtained in the HIT case. In the purely stratified case (run 7), the value of $a_{0}^{\|}$is slightly reduced compared to case 5 in the presence of both $\operatorname{RaS}\left(a_{0}^{\|} \approx 3.1\right)$. However, the value of $a_{0}^{\perp} \approx 1.4$ for case 7 is reduced by over an order of magnitude compared to case 5 . For the rotation-only run, $a_{0}$ is not very different compared to the HIT case, suggesting that the small scales are still dominated by turbulence, even though the large scales are highly anisotropic. This once again demonstrates that acceleration becomes progressively dominated, as Ro and Fr decrease, by the frequencies associated with rotation/buoyancy. As a consequence, the values of $a_{0}$ shown in Fig. 2 do not reflect only the small-scale structure of the flow, but also its global properties.

\section{B. Acceleration PDFs}

The analysis of acceleration variance can be generalized by considering the probability density functions (PDFs) of acceleration components. In HIT, similar to Eulerian velocity gradients, acceleration is also characterized by extremely large fluctuations reflected in broad tails of the PDF, which further broaden with increasing Reynolds number $[13,37]$. In this subsection, we study the effect of imposed RaS on the PDFs of acceleration, especially focusing on the long tails. Once again, we separate out the contributions $a_{\|}$and $a_{\perp}$. 

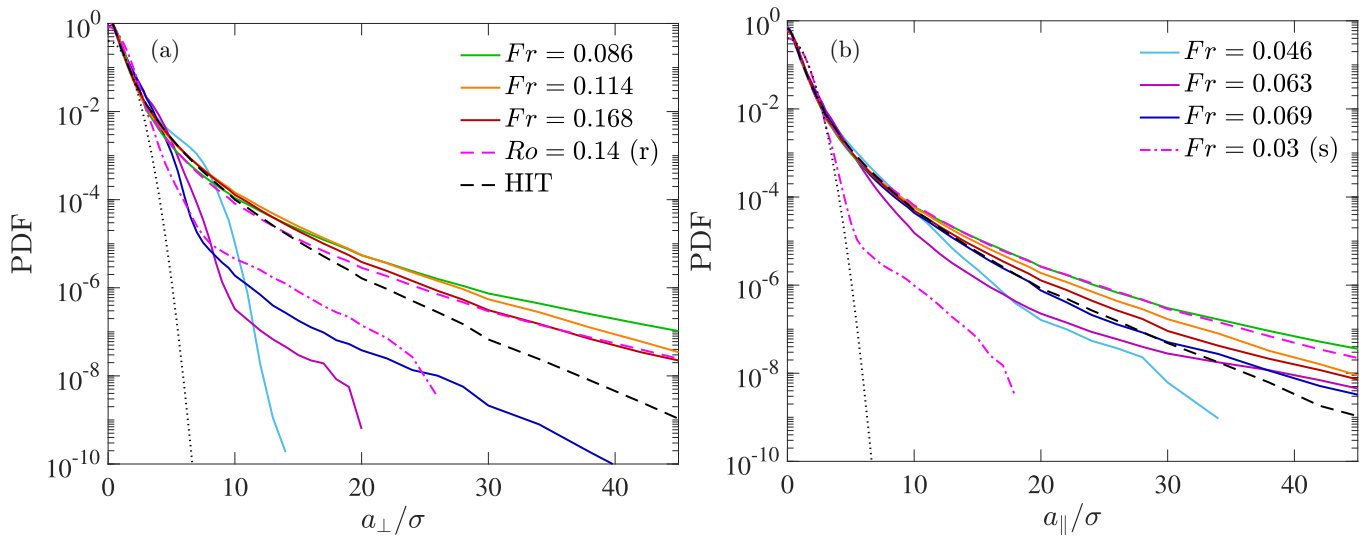

FIG. 3. Standardized probability density functions (PDFs) of (a) the perpendicular (horizontal) and (b) the parallel (vertical) components of acceleration, i.e., $a_{\perp}$ and $a_{\|}$, respectively. $\sigma$ denotes the corresponding standard deviation. The dotted line shows the standardized Gaussian distribution for comparison. The legend is split over two panels, but applies to each panel individually.

Figures 3(a) and 3(b), respectively, show the standardized PDFs of $a_{\perp}$ and $a_{\|}$for all the runs listed in Table III. Two very distinct behaviors can be observed once again. At moderate RaS (and for $R_{\mathrm{IB}}>1$ ) the PDFs of acceleration exhibit very broad tails, and in this sense, they differ only quantitatively from those obtained in the HIT case. This property is also reflected by the values of the kurtosis of the distributions, shown in Table III, which are all $\gtrsim 20$, even larger than the values obtained for the HIT case. The run corresponding to pure rotation also exhibits very high kurtosis of the acceleration. Note that the accuracy of the estimates of kurtosis indicated in the table are of the order of $10 \%$ or less, except for values marked with an asterisk, which exhibited very large fluctuations, leading to higher error bars for runs 3 and 8 (of about 20-30\%).

In contrast for the runs with strong $\mathrm{RaS}$ (with $R_{\mathrm{IB}}<1$ ), the tails of the PDFs are significantly suppressed. As shown in Table III, the corresponding kurtosis values are also quite small, although still larger than the Gaussian value of 3 . This is particularly clear for the horizontal component $a_{\perp}$ of acceleration, see Fig. 3(a), and to a lesser extent, for the vertical component $a_{\|}$[Fig. 3(b)]. The tendency of the PDFs to become narrower in runs with strong RaS appears to be a consequence of the strong stratification, as the PDFs of acceleration in the run with pure rotation are only weakly modified. In regimes dominated by waves, weak turbulence theory suggests that the velocity and acceleration should be close to Gaussian [52]. However, we observe that the kurtosis of acceleration components are significantly larger than the corresponding Gaussian value of 3 (with a stronger kurtosis for the vertical, than for the horizontal component of acceleration). This unexpected phenomenon can also be associated with the observation of bursts of vertical velocity, manifested by the large values of $K_{u_{\|}}$listed in Table II, particularly for runs 4-6. These bursts are related to the phenomenon observed in Ref. [34] in the case of purely stratified flows, in comparable domains of parameters. We expect that the mechanisms are similar in the presence of a relatively weak rotation $(N / f=5)$, as it is the case in the present study.

\section{Acceleration autocorrelations and frequency spectra}

Essential information on the motion of tracers can be obtained from the autocorrelation function of quantities fluctuating along Lagrangian trajectories. For example, the autocorrelation function can be used to determine relevant timescales and to form the frequency spectrum via Fourier transform [53]. Since our analysis is concerned with statistically stationary signals, the autocorrelation only depends on the chosen time lag. The autocorrelation $\rho^{a}(\tau)$ is defined as

$$
C^{a}(\tau)=\langle a(t+\tau) a(t)\rangle, \quad \rho^{a}(\tau)=C^{a}(\tau) / C^{a}(0),
$$



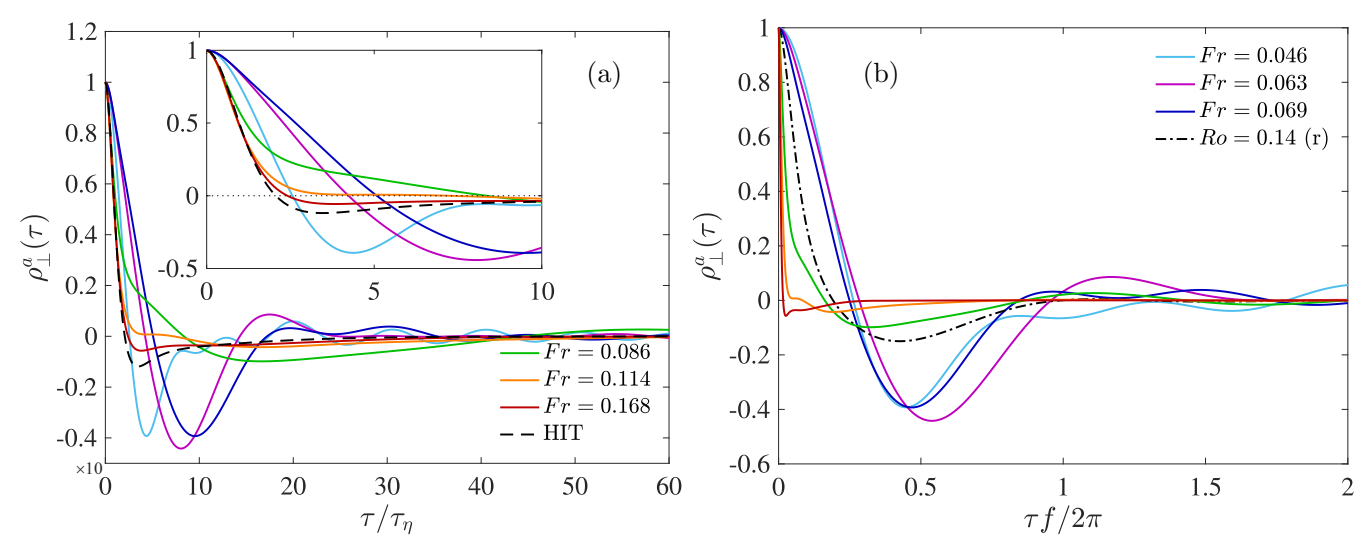

FIG. 4. Lagrangian autocorrelation of the perpendicular (horizontal) component of acceleration $\rho_{\perp}^{a}(\tau)$ against the time lag $\tau$ normalized by the Kolmogorov timescale $\tau_{\eta}$ in panel (a) and by the rotation period $2 \pi / f$ in panel (b). Inset in panel (a) shows a zoomed view at small-time lag. The legend is split over two panels, but applies to each panel individually.

where $\tau$ is the time lag and $C^{a}$ is the autocovariance. Statistical stationarity implies $\rho^{a}(-\tau)=$ $\rho^{a}(\tau)$ and also imposes that the integral of the acceleration autocorrelation must be zero, i.e., $\int_{0}^{\infty} \rho^{a}(\tau) d \tau=0$ [53]. The frequency spectrum of acceleration $E^{a}$, which is the Fourier transform of $C^{a}(\tau)$ can be defined as

$$
E^{a}(\omega)=\int_{0}^{\infty} C^{a}(\tau) \exp ^{-i \omega \tau} d \tau .
$$

By definition, the integral of the spectrum gives the variance of the signal. Yet again, we consider $a_{\perp}$ and $a_{\|}$separately.

\section{Perpendicular component}

Figure 4 shows the autororrelation function $\rho_{\perp}^{a}(\tau)$ for all the runs with RaS. In Fig. 4(a), the time lag is normalized with $\tau_{\eta}$, the Kolmogorov timescale characterizing the small-scale motion. We also include the HIT case (dashed line) and the pure rotation case (dash-dotted line) as a reference. For HIT, the acceleration autocorrelation is known to decay rapidly, becoming zero at $\tau \approx 2 \tau_{\eta}$ and thereafter becoming negative and slowly approaching zero again at very-large-time lags [54] —as readily seen in Fig. 4(a). Interestingly, we observe that the runs with relatively weak RaS, i.e., Fr $\geqslant 0.114$, and also the run with only rotation, behave very similarly to the HIT case [see inset of Fig. 4(a)], albeit with minor variation in the zero-crossing point. For the rot-strat run with $\mathrm{Fr}=0.086$, the autocorrelation function shows a similar behavior up to very-small-time lags, but thereafter deviates with the zero-crossing extending to $\tau \approx 8 \tau_{\eta}$. The large-time behavior is markedly different with very low frequency oscillations. However, for runs with strong RaS, i.e., $\mathrm{Fr} \leqslant 0.07$, a very different behavior is observed right from small-time lags. The autocorrelation shows strong oscillations which are eventually damped out at large-time lags. Furthermore, the zero-crossing point also is strongly dependent on Fr, moving to smaller time lags with decreasing Fr.

Plotting $\rho_{\perp}^{a}$ as a function of $\tau f / 2 \pi$, see Fig. 4(b), shows that the period of the damped oscillations is close to $2 \pi / f$ (with small but significant deviation). For comparison, we also show in Fig. 4(b) the function $\rho_{\perp}^{a}$ for the case of a purely rotating flow. Somewhat surprisingly, the tendency to oscillate at a frequency $\sim f$ is not as strong as in the case where both $\mathrm{RaS}$ are present. A similar trend was observed in Ref. [14]. We notice, however, that the stronger stratification ( $N=5 f$ in runs $1-6)$ implies the presence of faster waves than in a purely rotating flow at the same Ro number, involving 

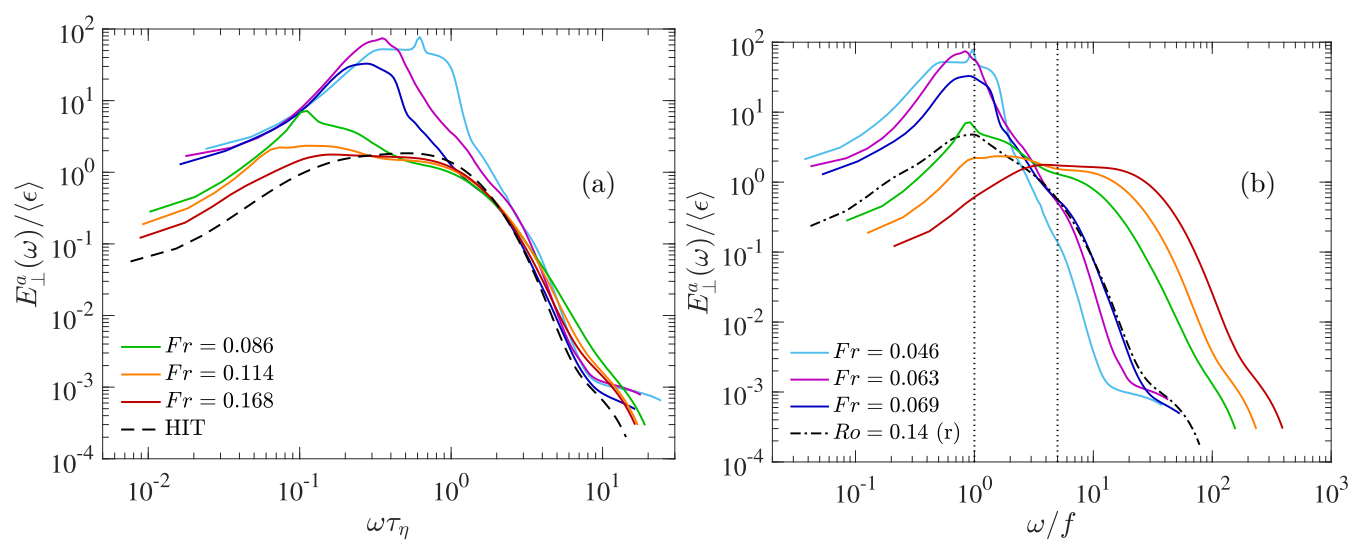

FIG. 5. Lagrangian frequency spectrum of the perpendicular (horizontal) component of acceleration $E_{\perp}^{a}(\omega)$ against the frequency $\omega$ normalized by the Kolmogorov timescale $\tau_{\eta}$ in panel (a) and by the rotation frequency $f$ in panel (b). The spectra are nondimensionalized using the mean kinetic energy dissipation rate $\langle\epsilon\rangle$. Vertical dotted lines in panel (b) correspond to frequencies $\omega=f$ and $N$ (note $N=5 f$ ). The legend is split over two panels, but applies to each panel individually.

in particular high values of $k_{\perp}$, since the dispersion law for inertia-gravity waves can be written as $k^{2} \omega_{I G}^{2}=N^{2} k_{\perp}^{2}+f^{2} k_{\|}[1]$.

To better identify the frequencies present in the autocorrelation function, it is apt to take its Fourier transform to obtain the frequency spectrum. Figure 5 shows the spectrum $E_{\perp}^{a}$. The spectra have been made dimensionless by dividing by the mean kinetic energy dissipation rate $\langle\epsilon\rangle$. In Fig. 5(a), the frequency is normalized by $\tau_{\eta}$, whereas in Fig. 5(b), we use the rotation frequency $f$. Note that the runs shown in Figs. 5(a) and 5(b) correspond to the same runs shown, respectively, in Figs. 4(a) and 4(b). For the HIT run in Fig. 5(a), Kolmogorov scaling would imply that the spectrum $E^{a}$ is constant in the inertial range (defined as $\tau_{\eta} / T_{E} \lesssim \omega \tau_{\eta} \lesssim 1$ ). While the validity of Kolmogorov's similarity hypotheses to Lagrangian statistics is debatable, DNS studies suggest that the above scaling law, i.e., $E^{a} \sim\langle\epsilon\rangle$, still may be approximately satisfied [55]. Consistent with this, we find that for our HIT run, the spectrum $E_{\perp}^{a}(\omega)$ is approximately flat over a limited range of values of $\omega$.

With addition of weak RaS, the changes in the structure of the spectrum appear to be relatively minor. In particular, for runs with Fr $\geqslant 0.086$, the differences in spectra, relative to the HIT case, only become significant for $\omega \tau_{\eta} \lesssim 0.2$. For the particular case of $\mathrm{Fr}=0.086$, a small peak emerges around $\omega \tau_{\eta} \approx 0.1$. With increasing strength of $\mathrm{RaS}$, the differences become more pronounced, with all the cases for $\mathrm{Fr} \leqslant 0.086$ now showing a prominent peak (which is relatively broad). This observation is consistent with the behavior of the autocorrelation function at small times, as observed in Fig. 4. In addition, the increase in values of $E_{\perp}^{a} /\langle\epsilon\rangle$ also corresponds to the strong increase in the value of $a_{0}$ for small Fr cases, as shown in Fig. 2 (for the perpendicular data points). Interestingly, the decay of spectra for all cases are somewhat similar at very large frequencies (and not very different from the HIT case). This possibly confirms that the role of turbulent eddies in the horizontal direction is still relevant as compared to the vertical direction, at least in the parameter range covered in this work (note once again the fact that $N / f=5$, plays an important role, since it renders the effect of rotation relatively weaker than that of stratification).

Figure 5(b) shows the spectra as a function of $\omega / f$. As expected, the peaks observed at low frequencies in Fig. 5(a), are all centered around $\omega / f \approx 1$. For the runs with strong RaS, the frequency corresponding to stratification, i.e., $\omega / f=N / f=5$, does not seem to play any particularly important role. 

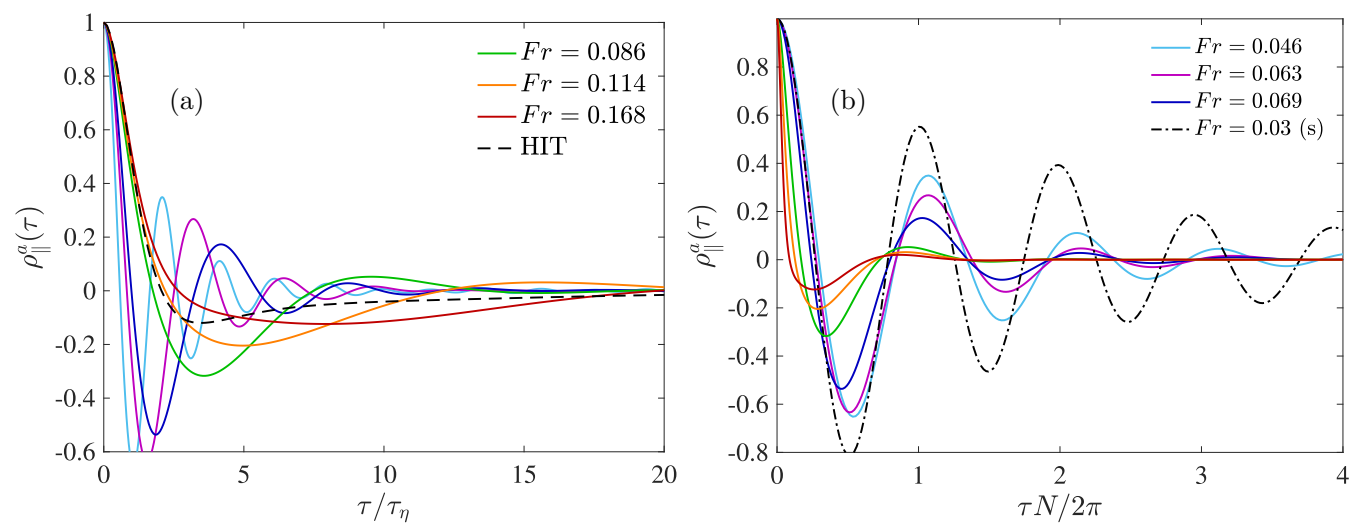

FIG. 6. Lagrangian autocorrelation of the parallel (vertical) component of acceleration $\rho_{\|}^{a}(\tau)$ against the time lag $\tau$ normalized by the Kolmogorov timescale $\tau_{\eta}$ in panel (a) and by the Brunt-Väisälä period $2 \pi / N$ in panel (b). The legend is split over two panels, but applies to each panel individually.

\section{Parallel component}

While the role of rotation was dominant in the perpendicular direction, we can in turn expect stratification to dominate in the parallel direction. In Figs. 6(a) and 6(b), we show the autocorrelation in the parallel direction $\rho_{\|}^{a}$ as a function of $\tau / \tau_{\eta}$ and of $\tau(N / 2 \pi)$, respectively. As it was the case for the perpendicular component of acceleration, the deviation of the autocorrelation function from the reference homogeneous isotropic case, shown as a dashed line, increases gradually when $\mathrm{Fr}$ decreases. However, contrary to $\rho_{\perp}^{a}, \rho_{\|}^{a}$ tends to decay significantly faster than in the HIT case when Fr diminishes. In addition, for all cases, overshooting oscillations are clearly visible, with a particularly strong amplitude for cases with $\mathrm{Fr} \leqslant 0.086$. While the zero-crossing of $\rho_{\|}^{a}$ for cases with weak RaS still appears to be close to $\tau \approx 2 \tau_{\eta}$ (with minor deviations), for cases with strong $\mathrm{RaS}$, the zero-crossing shifts to even smaller time lags of $\tau \approx 0.5 \tau_{\eta}$. This is in contrast to $\rho_{\perp}^{a}$, where the zero-crossing first shifted to higher time lags, and then moved to smaller time lags with decreasing Fr. Figure 6(b) clearly reveals that the period of the oscillations is equal to the Brunt-Väisälä period, $2 \pi / N$. This strongly suggests that the motion in the parallel direction is dominated by stratification, and that turbulence plays a much smaller role for the vertical motion (in comparison to horizontal motion, where even at strongest rotation rate, the role of turbulence still could not be ignored). For comparison, the autocorrelation function $\rho_{\|}^{a}$ is shown in Fig. 6(b) for the run with a strong stratification, $\mathrm{Fr}=0.03$. The tendency to oscillate is even stronger in this run, an effect amplified by the low value of Fr (and $R_{\mathrm{IB}}$ ) in this case (see Table II).

The corresponding frequency spectra $E_{\|}^{a}(\omega)$ are plotted as a function of $\omega \tau_{\eta}$ and of $\omega / N$ in Figs. 7(a) and 7(b), respectively. The spectra are again nondimensionalized by $\langle\epsilon\rangle$. Unlike in the case of $E_{\perp}^{a}$, we observe that deviations from the HIT run are already prominent even for runs with weak RaS. All spectra are characterized by the presence of a peak, with the peak becoming more sharp and prominent as Fr decreases. An inspection of spectra in Fig. 7(b) clearly shows that all these peaks correspond to $\omega / N \approx 1$, i.e., the respective Brunt-Väisälä frequencies, conforming with the dominant role of stratification in the vertical direction. Note the peak for the stratification-only run is even more pronounced. For runs with strong $\mathrm{RaS}$, i.e., $\mathrm{Fr} \leqslant 0.069$, a very minor enhancement of the spectra is visible in the band of frequencies $\omega / N \approx 0.2$ (or $\omega / f \approx 1$ ).

Another point to note is that even though the peaks in $E_{\|}^{a}$ become sharper with decreasing $\mathrm{Fr}$, their amplitudes do not vary much, in contrast to $E_{\perp}^{a}$, where the peaks vary by more than an order of magnitude. This can be explained by the variation of acceleration variance as seen in Fig. 2 . While the $a_{0}^{\perp}$ sharply shoots up for runs with Fr $\leqslant 0.069$, the $a_{0}^{\|}$only shows a minor variation in comparison. 

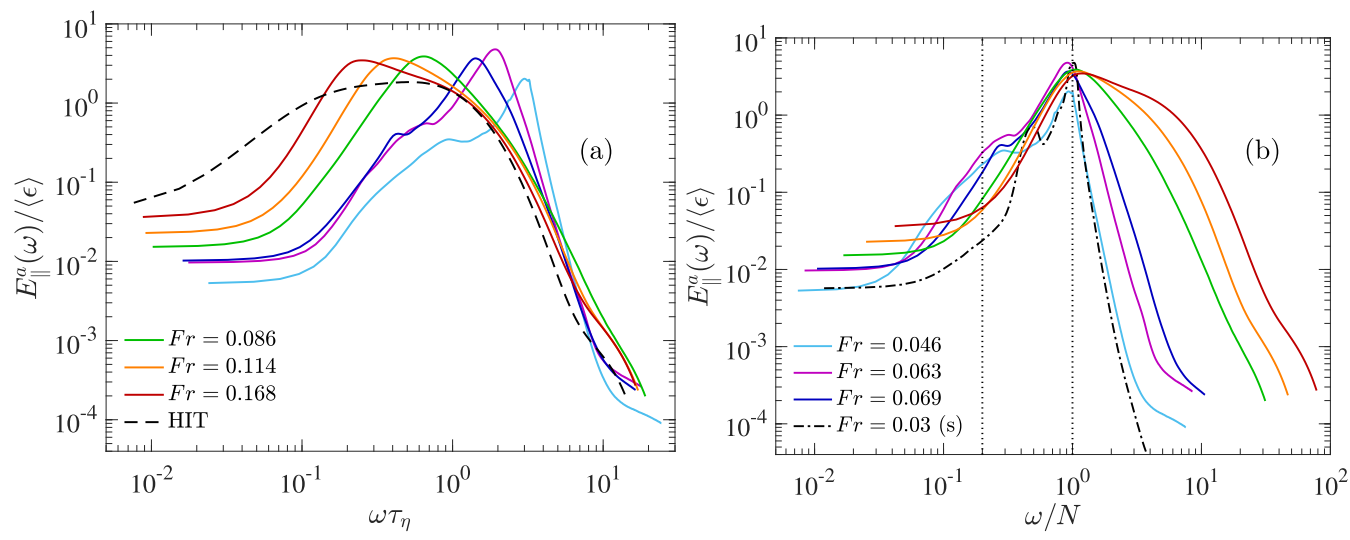

FIG. 7. Lagrangian frequency spectrum of the parallel (vertical) component of acceleration $E_{\|}^{a}(\omega)$ against the frequency $\omega$ normalized by the Kolmogorov timescale $\tau_{\eta}$ in panel (a) and by the Brunt-Väisälä frequency $N$ in panel (b). The spectra are nondimensionalized using the mean kinetic energy dissipation rate $\langle\epsilon\rangle$. Vertical dotted lines in panel (b) correspond to frequencies $\omega=f$ and $N$ (with $N=5 f$ ). The legend is split over two panels, but applies to each panel individually.

The results of this subsection complement the conclusions of Secs. IV A and IV B, as well as those of Sec. III, which were pointing to two qualitatively different regimes, dominated by waves for $R_{\mathrm{IB}}<1$, and by eddies $R_{\mathrm{IB}}>1$. Whereas in the latter regime, the variances and the PDFs of accelerations were showing only moderate deviations compared to HIT flow, Figs. 5(b) and 7(b) reveals the role of $N$ and $f$ in the horizontal and vertical motion closer to the transition, when $R_{\mathrm{IB}} \gtrsim$ $1\left(R_{\mathrm{IB}}=2.69\right.$ for run 3$)$. The transition to a wave-dominated regime for $R_{\mathrm{IB}}<1$ corresponds to the formation of peaks, which are much more intense [see Fig. 5(b)] or much sharper [see Fig. 7(b)], leading to a qualitatively very different dynamics.

\section{LAGRANGIAN VELOCITY AND DISPERSION STATISTICS}

The spread or dispersion of a material under the action of turbulence is of obvious importance and can be directly studied from the investigation of the Lagrangian velocity along trajectories. In this regard, we briefly summarize the classical theory below [7].

Single-particle dispersion is best understood by considering the mean-square displacement of a particle from its initial position. From Eq. (7), we can write for each direction:

$$
\left\langle Y_{i}^{2}(t)\right\rangle=\left\langle u_{i}(0)^{2}\right\rangle \int_{0}^{t} \int_{0}^{t} \rho^{u_{i}}\left(t^{\prime}, t^{\prime \prime}\right) d t^{\prime} d t^{\prime \prime}
$$

where $Y_{i}(t)=x_{i}(t)-x_{i}(0)$ is the displacement of the particle from its position $x_{i}(0)$ at $t=0$ to its position $x_{i}(t)$ at time $t$, and $\rho^{u_{i}}\left(t^{\prime}, t^{\prime \prime}\right)$ is the velocity autocorrelation. For statistical stationarity, the autocorrelation function at $t^{\prime}$ and $t^{\prime \prime}$ only depends on $\tau=\left|t^{\prime}-t^{\prime \prime}\right|$, allowing Eq. (11) to be rewritten as

$$
\left\langle Y_{i}^{2}(t)\right\rangle=2\left\langle u_{i}^{2}\right\rangle \int_{0}^{t}(t-\tau) \rho^{u_{i}}(\tau) d \tau
$$

Once again, we treat separately the horizontal and vertical components, as explained before. The expression for the dispersion, Eq. (12), simplifies in the limit of short and long times, respectively, to

$$
\begin{gathered}
\left\langle Y_{\perp, \|}^{2}(t)\right\rangle=\left\langle u_{\perp, \|}^{2}\right\rangle t^{2}, \quad \text { for } t \ll \tau_{K}, \\
\left\langle Y_{\perp, \|}^{2}(t)\right\rangle=D_{\perp, \|} t, \quad \text { for } \quad t \gg T_{L}^{\perp, \|},
\end{gathered}
$$



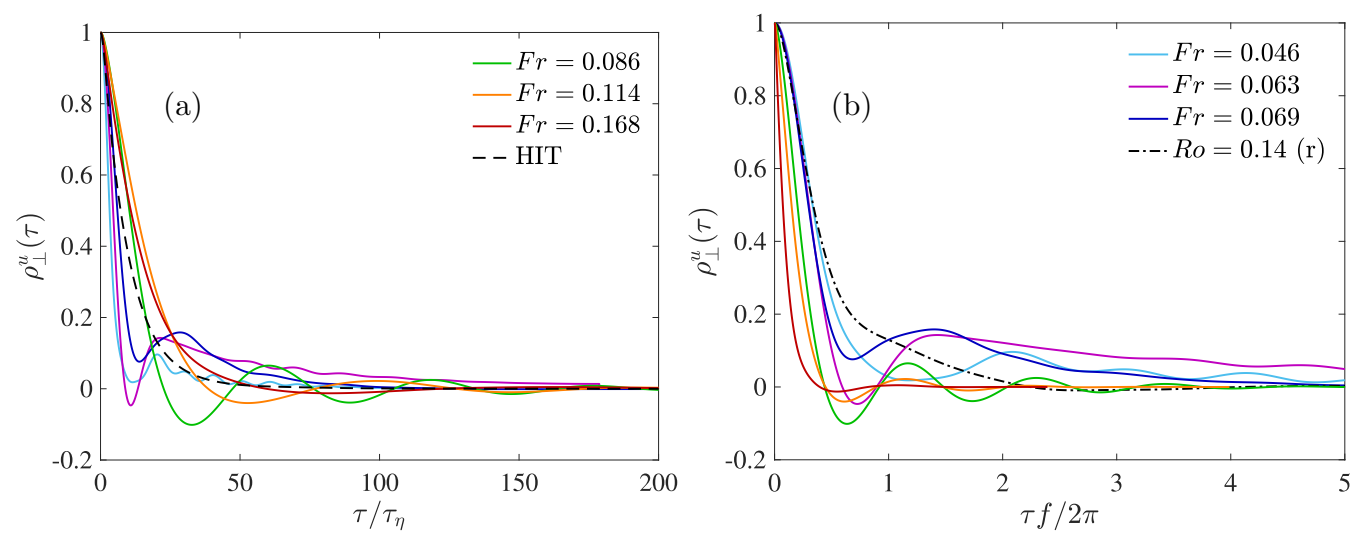

FIG. 8. Lagrangian autocorrelation $\rho_{\perp}^{u}(\tau)$ of the perpendicular (horizontal) component of the velocity, normalized by the Kolmogorov time scale $\tau_{\eta}$ in panel (a) and the rotation period $2 \pi / f$ in panel (b). The legend is split over two panels, but applies to each panel individually.

where $D_{\perp, \|}=2\left\langle u_{\perp, \|}^{2}\right\rangle T_{L}^{\perp, \|}$ are the diffusion coefficients and

$$
T_{L}^{\perp, \|}=\int_{0}^{\infty} \rho_{\perp, \|}^{u}(\tau) d \tau
$$

are the Lagrangian integral times. In the following subsections, we discuss separately the behavior of the autocorrelation functions, integral timescales and then the mean-square displacements.

\section{A. Velocity autocorrelations and integral timescales}

In this subsection, we investigate the behavior of the velocity autocorrelations and the corresponding integral timescales derived from them. Similar to the acceleration spectra, the velocity frequency spectra can be obtained through a Fourier transform of the velocity autocorrelations. However, the velocity spectra can also be simply derived from the acceleration spectra using the simple relation $E^{u}(\omega)=E^{a}(\omega) / \omega^{2}$ [53]. Consequently, we do not discuss the results for $E^{u}$ in this section (since they provide similar information as $E^{a}$ ). Instead, we have briefly summarized them in the Supplemental Material [42].

\section{Perpendicular component}

Figures 8(a) and 8(b) show the autocorrelation functions $\rho_{\perp}^{u}(\tau)$; similar to results for the perpendicular component of acceleration, the time lag $\tau$ is normalized first by $\tau_{\eta}$ and then by $f$. The correlation function $\rho_{\perp}^{u}$, plotted as a function of $\tau / \tau_{\eta}$, see Fig. 8(a), shows only weak deviations from the HIT case (shown in black dashed line) when RaS are moderate (runs 1-3). We recall that in the HIT case, the long time behavior of the velocity autocorrelation can be well represented by an exponential functional form, $\rho^{u} \sim \exp ^{-\tau / T_{L}}$ [12]. We notice that the runs with $\mathrm{RaS}$ are fundamentally different from the HIT run with appearance of oscillations resulting in negative values of the correlation function. These deviations become stronger with imposed RaS.

To better understand these oscillations, Fig. 8(b) shows the autocorrelation as a function of $\tau f / 2 \pi$. Although an oscillatory behavior can be seen in the runs with the highest values of RaS rates, the frequency is close to, but differs from $f$. However, $\rho_{\perp}^{u}$ in the flow with rotation only [shown in black dash-dotted line in Fig. 8(b)], appears to be monotonically decreasing (no oscillations). 

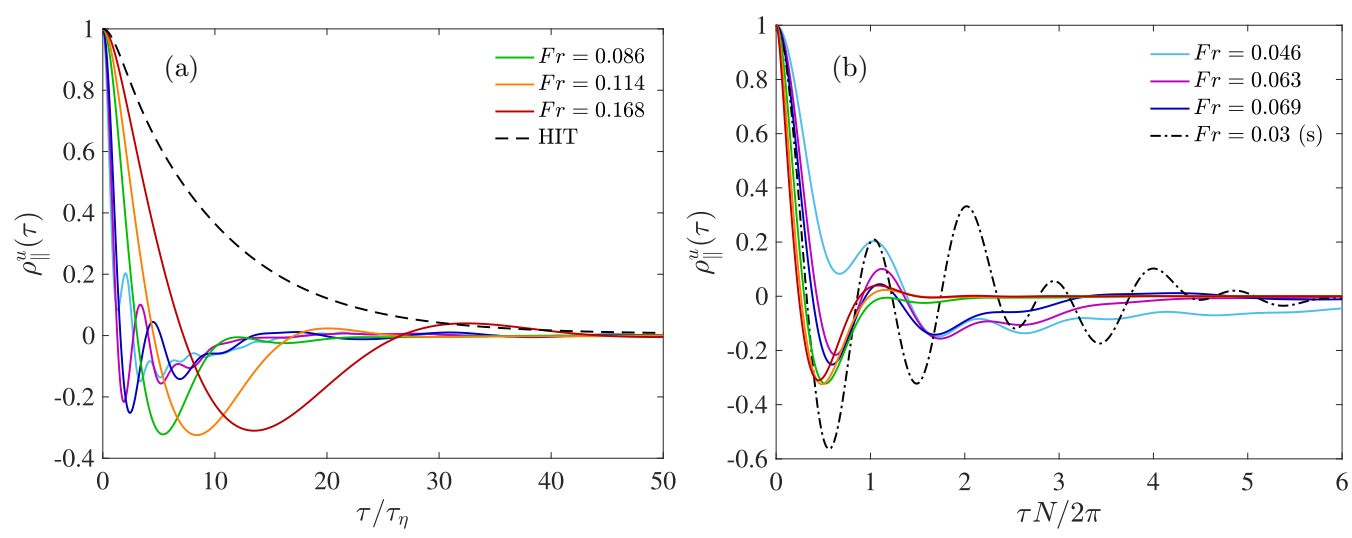

FIG. 9. Lagrangian autocorrelation $\rho_{\|}^{u}(\tau)$ of the parallel (vertical) component of the velocity. Similar normalizations as Fig. 6 are used. The legend is split over two panels but applies to each panel individually.

\section{Parallel component}

The autocorrelations and spectra corresponding to the parallel component of velocity are shown in Fig. 9. We first consider the autocorrelation $\rho_{\|}^{u}$ as a function of $\tau / \tau_{\eta}$ in Fig. 9(a). In all the cases with RaS, $\rho_{\|}^{u}$ strongly differs from the HIT case (indicated by a black dashed line). Two patterns are distinctly visible. First, all the autocorrelation functions decay rapidly. In terms of the dimensionless time $t / \tau_{\eta}$, the decay rate becomes increasingly larger when Fr decreases [especially in comparison to perpendicular component shown in Fig. 8(a)]. Second, the autocorrelations all overshoot to become strongly negative and show distinct oscillations. The primary period of these oscillations corresponds to the stratification frequency, as demonstrated by Fig. 9(b), which shows the autocorrelations as a function of $\tau N / 2 \pi$. In addition, Fig. 9(b) also shows that all cases superpose reasonably well for the initial decay of the autocorrelation, These results clearly demonstrate that stratification plays a dominant role in the vertical motion, and in comparison turbulence plays a weaker role. The fast dynamics in the vertical direction is dominated by the strong vertical gradients arising from the constraint that the vertical Froude number be of order unity [56], and leading to strong spatial and temporal intermittency, dissipation and mixing [57]. This is true, even for the runs at weak $\operatorname{RaS}(\mathrm{Fr} \gtrsim 0.08)$. A similar behavior was obtained for the vertical acceleration correlation function, see Sec. IV C. We notice that in the case of a purely stratified run, without any rotation, the autocorrelation function $\rho_{\|}^{u}$ clearly oscillates, with a modulation at twice the Brunt-Väisälä period.

\section{Integral timescales}

As highlighted by Eqs. (14) and (15), the Lagrangian integral timescale $T_{L}$, characterizes the particle dispersion at large times and is simply obtained by integrating the autocorrelation function: $T_{L}=\int_{0}^{\infty} \rho^{u} d \tau$. We begin by noticing that many of the correlation functions shown in Figs. 8 and 9 exhibit an oscillatory behavior. This makes the calculation of their integrals generally prone to statistical errors. As such, the values of $T_{L}^{\perp, \|}$, listed in Table IV, are subject to large relative errors, the more so as the values of $T_{L}^{\perp, \|}$ are small.

Over the range of Re considered here, the variation of $T_{L}^{\perp}$ is found to be rather weak at moderate $\operatorname{RaS}(\mathrm{Fr} \gtrsim 0.08)$. In particular, the ratio $T_{L}^{\perp} / \tau_{\eta}$, is of the order of 10 for the HIT run, and remains approximately constant for cases 1-4 and also for the run 8, which corresponds to a flow with rotation only. For cases 5 and 6 with strong RaS, the ratio sharply drops by a factor $\sim 2$, which can be attributed to a sharp decrease in the mean dissipation $\langle\epsilon\rangle$ (hence causing a sharp increase in $\tau_{\eta}$ ). The above observations are consistent with earlier results which also highlight a sharp transition to 
TABLE IV. Integral timescales of the velocity, $T_{L}^{\perp}$ and $T_{L}^{\|}$, respectively, based on the perpendicular (horizontal) component and parallel (vertical) component [see Eq. (15) for definition]. The integral timescales are also compared with the Kolmogorov time $\tau_{\eta}$, and of the Brunt-Väisälä period, $2 \pi / N$, for the vertical motion, and of the rotation period, $2 \pi / f$, for the horizontal motion. (A more detailed table is provided in the Supplemental Material [42].)

\begin{tabular}{lccccccccc}
\hline \hline Case & 0 & 1 & 2 & 3 & 4 & 5 & 6 & 7 & 8 \\
\hline Fr & $\infty$ & 0.168 & 0.114 & 0.086 & 0.069 & 0.063 & 0.045 & 0.030 & $\infty$ \\
Ro & $\infty$ & 0.840 & 0.570 & 0.430 & 0.345 & 0.315 & 0.225 & $\infty$ & 0.140 \\
$T_{L}^{\perp} / \tau_{\eta}$ & 11 & 14 & 14 & 10 & 12 & 4.1 & 5.8 & $\infty$ & 8.2 \\
$T_{L}^{\|} / \tau_{\eta}$ & 10 & 0.59 & 0.23 & 0.11 & $6.4 \times 10^{-2}$ & $2.7 \times 10^{-2}$ & $5.5 \times 10^{-2}$ & $3.6 \times 10^{-2}$ & 8.4 \\
$T_{L}^{\|} N /(2 \pi)$ & 0 & $2.0 \times 10^{-2}$ & $1.4 \times 10^{-2}$ & $1.1 \times 10^{-2}$ & $1.6 \times 10^{-2}$ & $9.0 \times 10^{-3}$ & $2.8 \times 10^{-2}$ & $1.2 \times 10^{-2}$ & 0 \\
\hline \hline
\end{tabular}

wave-dominated regime as $R_{\mathrm{IB}}$ significantly decreases. We note that for the run with pure stratification (case 7), the velocity autocorrelation in the horizontal direction converges extremely slowly to zero at large-time lags, pointing to an extremely large value of $T_{L}^{\perp}$. A similar behavior has been noticed in Ref. [19]. In this regard, the presence of rotation plays a crucial role. In particular, the dispersion relation and the structure of the eigenmodes, underlying the wave motion is strongly modified by rotation.

However, the integral time $T_{L}^{\|}$is substantially smaller than $T_{L}^{\perp}$ for all runs with stratification (cases 1-7). The ratio $T_{L}^{\|} / \tau_{\eta}$ is still around 10 for the HIT and purely rotating flows (cases 0 and 8 , respectively), but is greatly reduced for all runs with stratification. This can be evidently attributed to the strongly oscillating nature of the velocity autocorrelation functions for cases 1-7 [see Fig. 9(b)], which results in significant cancellation when calculating $T_{L}^{\|}$. The ratio $T_{L}^{\|} / \tau_{\eta}$ drops by more than an order of magnitude in going from HIT to case 1, which corresponds to the weakest RaS. Thereafter, with increasing strength of RaS, the ratio further decreases. Interestingly, the ratio $T_{L}^{\|} N / 2 \pi$ shows a much reduced variation, and remains in the range $0.01-0.02$ for all cases with stratification. This suggests that despite the strong oscillations in the autocorrelation the timescale, $T_{L}^{\|}$, is not strictly zero, but instead scales inversely with $N$, consistent with earlier predictions for purely stratified flows [58].

\section{B. Mean-square displacement}

Figure 10 shows the mean-square displacement for horizontal and vertical directions as a function of time (both axes normalized appropriately by Kolmogorov scales). At small times, a clear $t^{2}$ scaling is visible for all runs in both components, as expected from Eq. (13). The displacement in the horizontal direction is enhanced by RaS, whereas inhibited in the vertical direction. This can be readily explained by considering the variance of the components of the velocity $\left\langle v_{\perp}^{2}\right\rangle$ and $\left\langle v_{\|}^{2}\right\rangle$, as shown in Table II [see also Eq. (13)].

At long times, transition to a diffusive regime, given by Eq. (14), is expectedly seen in the HIT run [53], for both horizontal and vertical components. A similar behavior is also visible for runs with weak $\mathrm{RaS}(\mathrm{Fr} \gtrsim 0.08)$, when considering the horizontal component. In comparison, the growth of the vertical component at long times for these runs is significantly inhibited, nevertheless still showing a very slow approach to a diffusive regime. Our results are generally consistent with those obtained earlier for a comparable parameter range $(N / f=10, \mathrm{Fr} \approx 0.16)$ [59]. Note the rotationonly run, behaves like the HIT run, consistent with the small degree of anisotropy in both velocity and acceleration statistics as noted earlier.

In contrast, for runs with strong $\operatorname{RaS}(\mathrm{Fr} \lesssim 0.07)$, the growth of both horizontal and vertical components appears to be slower than a linear behavior. This is particularly clear for the vertical displacements $\left\langle Y_{\|}^{2}\right\rangle$, which appear to increase extremely slowly with time, if at all. In fact, the values 

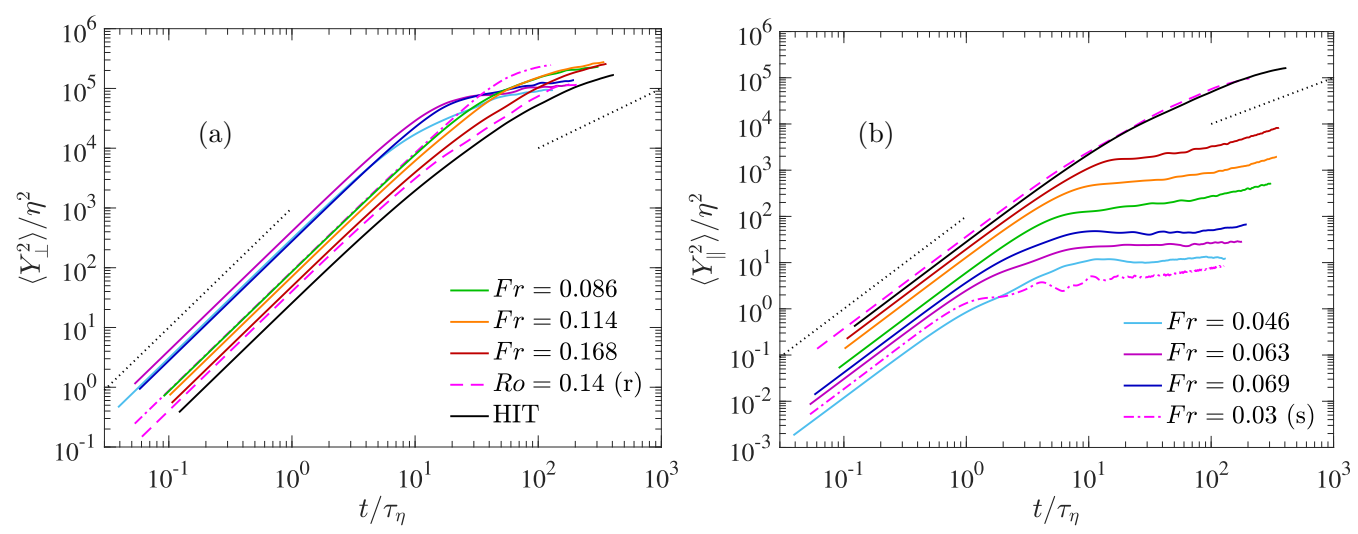

FIG. 10. Mean-square displacement as a function of time in the (a) perpendicular (horizontal) direction and (b) parallel (vertical) direction. All quantities normalized by Kolmogorov scales. The dotted lines represent slopes 2 and 1 at short and long times, respectively. The legend is split over two panels but applies to each panel individually.

of $\left\langle Y_{\|}^{2}\right\rangle / \eta^{2}$ remain small, of the order of $\sim 10$, over a very long time. The absence of a clear diffusive regime for $\left\langle Y_{\|}^{2}\right\rangle$ is arguably not very surprising, given the small values of $T_{\|}^{L}$ and $\left\langle v_{\|}^{2}\right\rangle$, hence of the diffusion coefficient $D=\left\langle v_{\|}^{2}\right\rangle T_{L}^{\|}$. Assuming that $T_{\|}^{L}$ is inversely proportional to $N$ (multiplied with a small prefactor), the expected diffusive behavior will likely be visible only after an extremely long time. Consistent with our own results, the near constancy of $\left\langle Y_{\|}^{2}\right\rangle$ was also reported in purely stratified flows [17-19].

Contrary to the very low values of $T_{L}^{\|}$for runs with strong RaS (see Table IV), the values of $T_{L}^{\perp}$ do not appear to be particularly small. This makes the lack of a clear diffusive regime for runs 4-6 for the horizontal displacement much more surprising. It has been noticed in the context of stratified flows, that the horizontal displacement could grow as $t^{2}$ [17]. Here, our results show that, in a regime dominated by waves (runs 4-6), the growth of $\left\langle Y_{\perp}^{2}\right\rangle$ is slower than $t^{1}$ over a long time. This suggests a strong interaction between the horizontal and vertical directions owing to the presence of rotation (and inertia-gravity waves), which was also visible in autocorrelations of acceleration and velocity. However, given the large values of $T_{L}^{\perp}$, it can be expected that the diffusive regime is ultimately reached at sufficiently longer times.

Finally, we note that the strong vertical drafts responsible for high kurtosis of $u_{\|}$(see Sec. III), and increased mixing efficiency [34], do not appear to cause an appreciable growth in $\left\langle Y_{\|}^{2}\right\rangle$ for the runs considered here. This is not inconsistent given the kurtosis is a normalized fourth-order moment, whereas $\left\langle Y_{\|}^{2}\right\rangle$ goes as the variance of vertical velocity and the integral scale $T_{L}^{\|}$, both of which are strongly suppressed. It is likely that at higher Reynolds numbers than considered here, the effect of the vertical drafts is felt more directly on the vertical displacement of fluid particles.

\section{DISCUSSION AND CONCLUSIONS}

Motivated by the well established effect of both rotation and stratification $(\mathrm{RaS})$ in numerous geophysical flows, and by the observation of Lagrangian tracers, such as buoys in the ocean [60], we have investigated the properties of particle trajectories in such flows. We have utilized direct numerical simulations of the Boussinesq equations in a periodic domain, with both stratification and the axis of rotation aligned in the vertical direction, in a statistically stationary regime maintained by stochastic forcing. We have focused on cases where the corresponding rotation and stratification frequencies, $f$ and $N$, respectively, are held in a fixed ratio $N / f=5$, as relevant in oceanographic situations [21]. The usual computer limitations have restricted the Reynolds numbers to approximately around 4000 in our runs, which are smaller than those observed in nature, but 
still large enough to allow for turbulence to adequately develop. The Froude numbers investigated are in the range $0.03 \lesssim \mathrm{Fr} \lesssim 0.2$, with corresponding Rossby number $\mathrm{Ro}=5 \mathrm{Fr}$, compatible with geophysical fluid flows. Our results illustrate the complex physical effects involved in a turbulent flow under the combined action of RaS. Whereas the corresponding physical effects have been recently investigated in an Eulerian context in many studies, we have documented the elementary properties of particle trajectories, in particular concerning acceleration, velocity and displacement statistics. Due to the imposed large-scale anisotropy, we differentiate between motion in the horizontal plane and in the vertical direction. We also appropriately compare with rotation-only and stratification-only runs and also with the well studied case of isotropic turbulence.

While the parameters Fr and Ro vary smoothly with the strength of imposed RaS, we observe a sharp change in the nature of the flow when $\mathrm{Fr} \lesssim 0.07$ for the runs considered, corresponding to a regime where waves appear to dominate over the nonlinearities, as also observed in Ref. [34] for stratification-only runs. An inspection of the Eulerian energy spectra (and the temperature spectra), shows a clear transition from a Kolmogorov $k^{-5 / 3}$ like behavior to a Bolgiano $k^{-11 / 5}$ like behavior at intermediate wave numbers. The transition also corresponds to the peak of intermittent disruption of shear layers, with a maximum of the kurtosis of the vertical velocity. In this context, our work extends the stratification-only results of [34]. Interestingly, we find that this transition to wavedominated regime can be given by a simple condition of $R_{\mathrm{IB}}<1$ based on the buoyancy Reynolds number $R_{\mathrm{IB}}$.

The effect of this transition is also evident in Lagrangian statistics. For acceleration statistics, the effect of imposed RaS appears very weak before the transition, suggesting the imposed anisotropy does not affect the small-scale isotropy significantly. The probability density functions (PDFs) of acceleration in both horizontal and vertical direction display wide tails very similar to isotropic turbulence, and the variance of acceleration can be approximately scaled with Kolmogorov variables. The Lagrangian autocorrelations and frequency spectra also appear to behave in a similar fashion, although a weak effect of $\mathrm{RaS}$ is still visible at low frequencies in the spectra (when $\left.R_{\mathrm{IB}} \gtrsim 1\right)$. However, the sharp transition at $R_{\mathrm{IB}} \lesssim 1$ leads to a very strong anisotropy between the horizontal and vertical directions, with the particle motion strongly dominated by RaS instead of turbulent eddies. The PDFs of acceleration show suppressed tails, and acceleration variance shows no clear scaling. Moreover, the autocorrelations and frequency spectra clearly reflect the dominant roles of frequencies $N$ and $f$.

On the other hand, Lagrangian velocity statistics are always affected by the imposed RaS. Anisotropy is already evident for weak RaS; however the degree of anisotropy becomes very strong with the said transition at $R_{\mathrm{IB}} \lesssim 1$. This is most readily seen in the integral timescale based on the autocorrelations from vertical velocity and hence also reflected in long time behavior of mean-square displacement of particles in the vertical direction. Similar effects had been observed in the case of purely stratified flows [19]. In such flows, it is known that stratification leads to the formation of horizontal layers, that inhibits the transport in the vertical direction. In the presence of both RaS, slanted layers appear [23] which somewhat inhibit transport in both the vertical and the horizontal directions.

It is interesting to consider that the transitional behavior observed in this work is possibly also linked to the enhanced vertical velocities that develop in strongly stably stratified turbulent flows, such as in the nocturnal planetary boundary layer [61], and warrant further examination. The resulting dispersion of Lagrangian particles must feel these hot spots of strong vertical velocity and strong local dissipation linked to localized shear instabilities, as also observed in the ocean. Several studies point to a local Richardson number, measuring stratification with respect to the internal shear associated with vertically sheared horizontal winds, to be mostly in the vicinity of the value for linear instability $[28,57,62]$. This leads to flows which are altogether close to criticality, and strongly anisotropic, intermittent and dissipative [57]. We expect that the observed similarity between the statistical properties of acceleration when the flow is dominated by eddies (for $R_{\mathrm{IB}}>1$ ), and those in HIT, may be very useful in modeling the dispersion of particles in turbulent flows in the presence of RaS. 
Whereas $R_{\mathrm{IB}}$ seems to provide a clear criterion to distinguish between the different regimes observed here, alternative ways [32] have been proposed to describe the transition between wavedominated flows, and flows where waves and eddies interact. Namely, it was found that the transition occurs around $R_{\mathcal{B}} \sim 10$, where $R_{\mathcal{B}}=\operatorname{ReFr}^{2}$. In this sense, the flows studied here are all in the regime of interacting waves and eddies, although run 6 is very close to the transition toward a wave-dominated flow.

We conclude by mentioning that many interesting issues remain to be addressed for particle motion in such flows. For example, the problem of separation of pairs, or of clusters of particles, both forward and backward in time $[63,64]$ or considering the effects of particle inertia or molecular diffusion. In addition, we stress that the picture we have drawn is based on runs at a relative moderate Reynolds numbers. Exploring higher Reynolds numbers, and also higher Prandtl/Schmidt numbers [65], along with different values of $N / f$ will help in better understanding the parameter space and how the results from current work translate to practical geophysical applications.

\section{ACKNOWLEDGMENTS}

The computing resources utilized in this work were provided by PSMN at the Ecole Normale Superieure de Lyon. D. Buaria and A. Pumir acknowledge support from the European Highperformance Infrastructure in Turbulence (EuHIT) program and the Max Planck Society. A. Pumir acknowledges additional support from the IDEXLYON project (Contract No. ANR-16-IDEX-0005) under University of Lyon auspices. R. Marino acknowledges support from the program PALSE (Programme Avenir Lyon Saint-Etienne) and IMPULSION of the University of Lyon, in the framework of the program Investissements d'Avenir (ANR-11-IDEX-0007), from the Inge'LySE (Lyon-Saint-Etienne) and from ERCOFTAC. A. Pouquet gratefully acknowledges support from Laboratory for Atmospheric and Space Physics (LASP), in particular Bob Ergun, at the University of Colorado, Boulder.

[1] J. Pedlosky, Geophysical Fluid Dynamics (Springer, Berlin, 1979).

[2] J. B. Weiss and A. Provenzale, Transport and Mixing in Geophysical Flows (Springer, Berlin, 2007), Vol. 744.

[3] J. C. Wyngaard, Atmospheric turbulence, Annu. Rev. Fluid Mech. 24, 205 (1992).

[4] A. Stohl, S. Eckhardt, C. Forster, P. James, and N. Spichtinger, On the pathways and timescales of intercontinental air pollution transport, J. Geophys. Res.: Atmos. 107, ACH-6 (2002).

[5] R. A. Shaw, Particle-turbulence interactions in atmospheric clouds, Ann. Rev. Fluid Mech. 35, 183-227 (2003).

[6] J. S. Guasto, R. Rusconi, and R. Stocker, Fluid mechanics of planktonic microorganisms, Annu. Rev. Fluid Mech. 44, 373 (2012).

[7] A. S. Monin and A. M. Yaglom, Statistical Fluid Mechanics (MIT Press, Cambridge, MA, 1971), Vol. 1.

[8] D. Buaria, P. K. Yeung, and B. L. Sawford, A Lagrangian study of turbulent mixing: Forward and backward dispersion of molecular trajectories in isotropic turbulence, J. Fluid Mech. 799, 352 (2016).

[9] A. Pumir and M. Wilkinson, Collisional aggregation due to turbulence, Annu. Rev. Condens. Matter Phys. 7, 141 (2016).

[10] P. A. Davidson, Turbulence in Rotating, Stratified and Electrically Conducting Fluids (Cambridge University Press, Cambridge, UK, 2013).

[11] B. Sawford, Turbulent relative dispersion, Annu. Rev. Fluid Mech. 33, 289 (2001).

[12] P. K. Yeung, Lagrangian investigations of turbulence, Ann. Rev. Fluid Mech. 34, 115 (2002).

[13] F. Toschi and E. Bodenschatz, Lagrangian properties of particles in turbulence, Annu. Rev. Fluid Mech. 41, 375 (2009).

[14] L. DelCastello and H. J. H. Clercx, Lagrangian Acceleration of Passive Tracers in Statistically Steady Rotating Turbulence, Phys. Rev. Lett. 107, 214502 (2011). 
[15] L. DelCastello and H. J. H. Clercx, Lagrangian velocity autocorrelations in statistically steady rotating turbulence, Phys. Rev. E 83, 056316 (2011).

[16] A. Naso and F. S. Godeferd, Statistics of the perceived velocity gradient tensor in a rotating turbulent flow, New J. Phys. 14, 125002 (2012).

[17] M. van Artrijk, H. J. H. Clercx, and K. B. Winters, Single-particle, particle-pair, and multiparticle dispersion of fluid particles in forced stably stratified turbulence, Phys. Fluids 20, 025104 (2008).

[18] G. Brethouwer and E. Lindborg, Numerical study of vertical dispersion by stratified turbulence, J. Fluid Mech. 631, 149 (2009).

[19] N. E. Sujovolsky, P. D. Mininni, and M. P. Rast, Single-particle dispersion in stably stratified turbulence, Phys. Rev. Fluids 3, 034603 (2018).

[20] A. C. N. Garabato, K. L. Polzin, B. A. King, K. J. Heywood, and M. Visbeck, Widespread intense turbulent mixing in the southern ocean, Science 303, 210 (2004).

[21] M. Nikurashin, G. K. Vallis, and A. Adcroft, Routes to energy dissipation for geostrophic flows in the southern ocean, Nat. Geosci. 6, 48 (2012).

[22] H. van Haren and L. Gostiaux, Convective mixing by internal waves in the Puerto Rico Trench, J. Mar. Res. 74, 161 (2016).

[23] R. Marino, P. D. Mininni, D. Rosenberg, and A. Pouquet, Emergence of helicity in rotating stratified turbulence, Phys. Rev. E 87, 033016 (2013).

[24] A. Pouquet and R. Marino, Geophysical Turbulence and the Duality of the Energy Flow Across Scales, Phys. Rev. Lett. 111, 234501 (2013).

[25] G. Haller, Lagrangian coherent structures, Annu. Rev. Fluid Mech. 47, 137 (2015).

[26] G. Ivey, K. Winters, and J. Koseff, Density stratification, turbulence but how much mixing? Ann. Rev. Fluid Mech. 40, 169 (2008).

[27] E. Deusebio, P. Augier, and E. Lindborg, Third-order structure functions in rotating and stratified turbulence: A comparison between numerical, analytical and observational results, J. Fluid Mech. 755, 294 (2014).

[28] D. Rosenberg, A. Pouquet, R. Marino, and P. D. Mininni, Evidence for Bolgiano-Obukhov scaling in rotating-stratified turbulence using high-resolution direct numerical simulations, Phys. Fluids 27, 055105 (2015).

[29] E. Lindborg and A. V. Mohanan, A two-dimensional toy model for geophysical turbulence, Phys. Fluids 29, 111114 (2017).

[30] M. L. Waite, Random forcing of geostrophic motion in rotating stratified turbulence, Phys. Fluids 29 126602 (2017).

[31] M.C. Gregg, E.A. D’Asaro, J.J. Riley, and E. Kunze, Mixing efficiency in the ocean, Ann. Rev. Marine Sci. 10, 443 (2018).

[32] A. Pouquet, D. Rosenberg, R. Marino, and C. Herbert, Scaling laws for mixing and dissipation in unforced rotating stratified turbulence, J. Fluid Mech. 844, 519 (2018).

[33] C. Rorai, P. D. Mininni, and A. Pouquet, Turbulence comes in bursts in stably stratified flows, Phys. Rev. E 89, 043002 (2014).

[34] F. Feraco, R. Marino, A. Pumir, L. Primavera, P. D. Mininni, A. Pouquet, and D. Rosenberg, Vertical drafts and mixing in stratified turbulence: Sharp transition with the Froude number, Europhys. Lett. 123, 44002 (2018).

[35] R. Marino, P. D. Mininni, D. Rosenberg, and A. Pouquet, Inverse cascade in rotating stratified turbulence: Fast growth of large scales, Europhys. Lett. 102, 44006 (2013).

[36] A. Pouquet, D. Rosenberg, J. Stawarz, and R. Marino, Helicity dynamics, inverse, and bidirectional cascades in fluid and magnetohydrodynamic turbulence: A brief review, Earth Space Sci. 6, 351 (2019).

[37] D. Buaria, A. Pumir, E. Bodenschatz, and P. K. Yeung, Extreme velocity gradients in turbulent flows, New J. Phys. 21, 043004 (2019).

[38] R. Marino, D. Rosenberg, C. Herbert, and A. Pouquet, Interplay of waves and eddies in rotating stratified turbulence and the link with kinetic-potential energy partition, Europhys. Lett. 112, 49001 (2015).

[39] G. Brethouwer, P. Billant, E. Lindborg, and J. M. Chomaz, Scaling analysis and simulations of strongly stratified flows, J. Fluid Mech. 585, 343 (2007). 
[40] T. Ishihara, T. Gotoh, and Y. Kaneda, Study of high-Reynolds number isotropic turbulence by direct numerical simulations, Ann. Rev. Fluid Mech. 41, 165 (2009).

[41] P. D. Mininni, D. Rosenberg, R. Reddy, and A. Pouquet, A hybrid mpi-openmp scheme for scalable parallel pseudospectral computations for fluid turbulence, Parallel Comput. 37, 316 (2011).

[42] See Supplemental Material at http://link.aps.org/supplemental/10.1103/PhysRevFluids.5.064801 for additional details on stationarity of runs, Lagrangian velocity spectra and integral time scales.

[43] M. A. T. van Hinsberg, J. H. M. ten Thije Boonkkamp, F. Toschi, and H. J. H. Clercx, Optimal interpolation schemes for particle tracking in turbulence, Phys. Rev. E 87, 043307 (2013).

[44] D. Buaria and P. K. Yeung, A highly scalable particle tracking algorithm using partitioned global address space (PGAS) programming for extreme-scale turbulence simulations, Comput. Phys. Commun. 221, 246 (2017).

[45] E. Lindborg, The energy cascade in a strongly stratified fluid, J. Fluid Mech. 550, 207 (2006).

[46] M. Verma, A. Kumar, and A. Pandey, Phenomenology of buoyancy-driven turbulence: Recent results, New J. Phys. 19, 025012 (2017).

[47] R. Bolgiano, Turbulent spectra in a stably stratified atmosphere, J. Geophys. Res. 64, 2226 (1959).

[48] A. M. Obukhov, Effect of the Archimedean forces on the structure of the temperature field in a turbulent flow, Dokl. Akad. Nauk SSSR 125, 1246 (1959).

[49] Y. Kimura and J. R. Herring, Energy spectra of stably stratified turbulence, J. Fluid Mech. 698, 19 (2012).

[50] J. D. Wilson and B. L. Sawford, Review of Lagrangian stochastic models for trajectories in the turbulent atmosphere, Bound.-Layer Met. 78, 191 (1996).

[51] B. L. Sawford, P. K. Yeung, M. S. Borgas, P. Vedula, A. La Porta, A. M. Crawford, and E. Bodenschatz, Conditional and unconditional acceleration statistics in turbulence, Phys. Fluids 15, 3478 (2003).

[52] S. Nazarenko, Wave Turbulence (Springer-Verlag, 2011).

[53] P. K. Yeung and S. B. Pope, Lagrangian statistics from direct numerical simulations of isotropic turbulence, J. Fluid Mech. 207, 531 (1989).

[54] P. K. Yeung, S. B. Pope, E. A. Kurth, and A. G. Lamorgese, Lagrangian conditional statistics, acceleration and local relative motion in numerically simulated isotropic turbulence, J Fluid Mech 582, 399 (2007).

[55] B. L. Sawford and P. K. Yeung, Kolmogorov similarity scaling for one-particle Lagrangian statistics, Phys. Fluids 23, 091704 (2011).

[56] P. Billant and J.-M. Chomaz, Self-similarity of strongly stratified inviscid flows, Phys. Fluids 13, 1645 (2001).

[57] A. Pouquet, D. Rosenberg, and R. Marino, Linking dissipation, anisotropy and intermittency in rotating stratified turbulence at the threshold of linear shear instabilities, Phys. Fluids 31, 105116 (2019).

[58] E. Lindborg and G. Brethouwer, Vertical dispersion by stratified turbulence, J. Fluid Mech. 614, 303 (2008).

[59] C. Cambon, F. S. Godeferd, F. C. G. A. Nicolleau, and J. C. Vassilicos, Turbulent diffusion in rapidly rotating flows with and without stable stratification, J. Fluid Mech. 499, 231 (2004).

[60] A. C. Poje, T. M. Özgökmen, D. J. Bogucki, and A. D. Kirwan, Jr., Evidence of a forward energy cascade and Kolmogorov self-similarity in submesoscale ocean surface drifter observations, Phys. Fluids 29, 020701 (2017).

[61] D. H. Lenschow, M. Lothon, S. D. Mayor, P. P. Sullivan, and G. Canut, A comparison of higher-order vertical velocity moments in the convective boundary layer from Lidar with in situ measurements and Large-Eddy Simulation, Bound. Lay. Met. 143, 107 (2012).

[62] W. D. Smyth, J. D. Nash, and J. N. Moum, Self-organized criticality in geophysical turbulence, Sci. Rep. 9, 3747 (2019).

[63] D. Buaria, B. L. Sawford, and P. K. Yeung, Characteristics of backward and forward two-particle relative dispersion in turbulence at different Reynolds numbers, Phys. Fluids 27, 105101 (2015).

[64] H. Xu, A. Pumir, and E. Bodenschatz, Lagrangian view of time irreversibility of fluid turbulence, Sci. China Phys. Mech. Astron. 59, 614702 (2015).

[65] D. Buaria, M. P. Clay, K. R. Sreenivasan, and P. K. Yeung, Turbulence is an ineffective mixer when Schmidt numbers are large, arXiv:2004.06202 (2020). 